\title{
Improved Nearness Research
}

\author{
Dieter Leseberg \\ Institut für Mathematik, Freie Universität, Berlin, Germany \\ Email: leseberg@zedat.fu-berlin.de
}

Received 20 August 2014; revised 26 September 2014; accepted 15 October 2014

Copyright (C) 2014 by author and Scientific Research Publishing Inc.

This work is licensed under the Creative Commons Attribution International License (CC BY).

http://creativecommons.org/licenses/by/4.0/

c) (i) Open Access

\begin{abstract}
In the realm of Bounded Topology we now consider supernearness spaces as a common generalization of various kinds of topological structures. Among them the so-called Lodato spaces are of significant interest. In one direction they are standing in one-to-one correspondence to some kind of topological extensions. This last statement also holds for contiguity spaces in the sense of Ivanova and Ivanov, respectively and moreover for bunch-determined nearness spaces as Bentley has shown in the past. Further, Doîtchînov proved that the compactly determined Hausdorff extensions of a given topological space are closely connected with a class of supertopologies which he called $b$-supertopologies. Now, the new class of supernearness spaces-called paranearness spaces-generalize all of them, and moreover its subclass of clan spaces is in one-to-one correspondence to a certain kind of symmetric strict topological extension. This is leading us to one theorem which generalize all former mentioned.
\end{abstract}

\section{Keywords}

Set-Convergence, Supertopological Space, Lodato Space, Contiguity Space, Nearness, Paranearness

\section{Basic Concepts}

As usual $\underline{P} X$ denotes the power set of a set $X$, and we use $\mathcal{B}^{X} \subset \underline{P} X$ to denote a collection of bounded subsets of $X$, also known as $\underline{B}$-sets, i.e. $\mathcal{B}^{X}$ has the following properties:

$\left(\mathrm{b}_{1}\right) \varnothing \in \mathcal{B}^{X}$

(b) $B_{2} \subset B_{1} \in \mathcal{B}^{X}$ imply $B_{2} \in \mathcal{B}^{X}$;

(b) $x \in X$ implies $\{x\} \in \mathcal{B}^{X}$.

Then, for $\underline{B}$-sets $\mathcal{B}^{X}, \mathcal{B}^{Y}$ a function $f: X \rightarrow Y$ is called bounded iff $f$ satisfies (b), i.e.

(b) $\left\{f[B]: B \in \mathcal{B}^{X}\right\} \subset \mathcal{B}^{Y}$.

Definition 1.1 For a set $X$, we call a triple $\left(X, \mathcal{B}^{X}, N\right)$ consisting of $X, \underline{B}$-set $\mathcal{B}^{X}$ and an operator $N: \mathcal{B}^{X} \rightarrow \underline{P}(\underline{P}(\underline{P} X))$ a prehypernear space iff the following axioms are satisfied, i.e. 
$\left(\mathrm{hn}_{1}\right) \quad B \in \mathcal{B}^{X}$ and $\rho_{2} \ll \rho_{1} \in N(B)$ imply $\rho_{2} \in N(B)$, where $\rho_{2} \ll \rho_{1}$ iff $\forall F_{2} \in \rho_{2} \exists F_{1} \in \rho_{1} F_{2} \supset F_{1}$;

$\left(\mathrm{hn}_{2}\right) \quad B \in \mathcal{B}^{X}$ implies $\mathcal{B}^{X} \notin N(B) \neq \varnothing$;

$\left(\mathrm{hn}_{3}\right) \quad \rho \in N(\varnothing)$ implies $\rho=\varnothing$;

$\left(\mathrm{hn}_{4}\right) \quad x \in X$ implies $\{\{x\}\} \in N(\{x\})$.

If $\rho \in N(B)$ for some $B \in \mathcal{B}^{X}$, then we call $\rho$ a $B$-near collection in $N$. For prehypernear spaces $\left(X, \mathcal{B}^{X}, N\right),\left(Y, \mathcal{B}^{X}, M\right)$ a bounded function $f: X \rightarrow Y$ is called a hypernear map, shortly hn-map iff it satisfies (hn), i.e.

(hn) $B \in \mathcal{B}^{X}$ and $\rho \in N(B)$ imply $\{f[F]: F \in \rho\}=: f \rho \in N(f[B])$; a sected hn-map, shortly shn-map iff it satisfies (shn), i.e.

(shn) $B \in \mathcal{B}^{X}$ and $\rho \in N(B)$ imply $\sec f(\sec \rho) \in N(f[B])$ with $\sec \rho:=\{T \subset X: \forall F \in \rho F \cap T \neq \varnothing\}$ and $f(\mathcal{A}):=\{D \subset Y: \exists A \in \mathcal{A} D \supset f[A]\}$.

Remark 1.2 Note, that shn-maps between prehypernear spaces are always hn-maps. We denote by $\mathrm{PHN}^{\bullet}$ respectively PHN the corresponding categories.

Examples 1.3 (i) For a prenearness space $(X, \xi)$ ([1]) let $\mathcal{B}^{X}$ be $\underline{B}$-set. Then we consider the triple $\left(X, \mathcal{B}^{X}, N_{\xi}\right)$ where $N_{\xi}(\varnothing):=\{\varnothing\}$ and $N_{\xi}(B):=\{\rho \subset \underline{P} X:\{B\} \cup \rho \in \xi\}$, otherwise.

(ii) For a $b$-filter space $\left(X, \mathcal{B}^{X}, \tau\right)$ ([2]) we consider the triple $\left(X, \mathcal{B}^{X}, N_{\tau}\right)$, where for each $B \in \mathcal{B}^{X} N_{\tau}$ is defined by setting: $N_{\tau}(B):=\{\rho \subset \underline{P} X: \exists \mathcal{F} \in \operatorname{FIL}(X)(\mathcal{F} \times \mathcal{F} \in \tau(B)$ and $\rho \subset \sec \mathcal{F})\}$;

(iii) For a set-convergence space $\left(X, \mathcal{M}^{X}, q\right)$ ([3] we consider the triple $\left(X, \mathcal{M}^{X}, N_{q}\right)$, where for each $\mathcal{B} \in \mathcal{M}^{X} N_{q}$ is defined by setting: $N_{q}(B):=\{\rho \subset \underline{P} X: \exists \mathcal{F} \in \operatorname{FIL}(X)(\rho \subset \sec \mathcal{F}$ and $\mathcal{F} q B)\}$;

(iv) For a generalized convergence space $(X, q)$ [4], we consider the triple $\left(X, \mathcal{D}(X), N^{q}\right)$, where

$$
\mathcal{D}(X):=\{\varnothing\} \cup\{\{x\}: x \in X\} \text { and } N^{q}(\{x\}):=\{\rho \subset \underline{P} X: \exists \mathcal{F} \in \operatorname{FIL}(X)(\rho \subset \sec \mathcal{F} \text { and } \mathcal{F} q x)\}
$$

for $x \in X$ with $N^{q}(\varnothing):=\{\varnothing\}$; alternately we look at the following triple $\left(X, \underline{P} X,{ }^{q} N\right)$, where

$$
{ }^{q} N(B):=\{\rho \subset \underline{P} X: \exists x \in B \exists \mathcal{F} \in \operatorname{FIL}(X)(\rho \subset \sec \mathcal{F} \text { and } \mathcal{F} q x)\} \text { for } \mathcal{B} \neq \varnothing,
$$

and ${ }^{a} N(\varnothing):=\{\varnothing\}$;

(v) For a $\breve{C}$ ech-closure space $(X,-)$ ([5]) let $\mathcal{B}^{X}$ be $\underline{B}$-set. Then we consider the triple $\left(X, \mathcal{B}^{X}, N^{-}\right)$ with $N^{-}(B):=\{\rho \subset \underline{P} X: B \in \sec \{\bar{F}: F \in \rho\}\}$ for each $B \in \mathcal{B}^{X}$;

(vi) For a $b$-proximity space $\left(X, \mathcal{M}^{X}, \delta\right)$ ([6]) we consider the triple $\left(X, \mathcal{M}^{X}, N_{\delta}\right)$, where

$$
N_{\delta}(B):=\{\rho \subset \underline{P} X: \rho \subset \delta(B)\}
$$

for each $\mathcal{B} \in \mathcal{M}^{X}$ with $\delta(B):=\{A \subset X: B \delta A\}$;

(vii) For a neighborhood space $\left(X, \mathcal{M}^{X}, \Theta\right)$ ([6]) we consider the triple $\left(X, \mathcal{M}^{X}, N_{\Theta}\right)$, where for each $\mathcal{B} \in \mathcal{M}^{X} N_{\Theta}(B):=\{\rho \subset \underline{P} X: \rho \subset \sec \Theta(B)\}$.

Remark 1.4 In preparing the next two important examples we give the following definitions.

Definitions 1.5 TEXT denote the category, whose objects are triples $E:=\left(e, \mathcal{B}^{X}, Y\right)$-called topological extensions-where $X:=\left(X, c l_{X}\right), Y:=\left(Y, c l_{Y}\right)$ are topological spaces (given by closure operators) with $\underline{B}$-set $\mathcal{B}^{X}$ and $e: X \rightarrow Y$ is a function satisfying the following conditions:

$\left(t x_{1}\right) \quad A \in \underline{P} X$ implies $c l_{X}(A)=e^{-1}\left[c l_{Y}(e[A])\right]$, where $e^{-1}$ denotes the inverse image under $e$;

$\left(t x_{2}\right) c l_{Y}(e[X])=Y$, which means that the image of $X$ under $e$ is dense in $Y$.

Morphisms in TEXT have the form $(f, g):\left(e, \mathcal{B}^{X}, Y\right) \rightarrow\left(e^{\prime}, \mathcal{B}^{X^{\prime}}, Y^{\prime}\right)$, where $f: X \rightarrow X^{\prime}, g: Y \rightarrow Y^{\prime}$ are continuous maps such that $f$ is bounded, and the following diagram commutes:

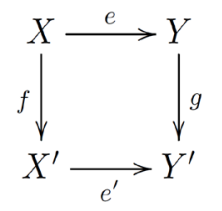

If $(f, g):\left(e, \mathcal{B}^{X}, Y\right) \rightarrow\left(e^{\prime}, \mathcal{B}^{X^{\prime}}, Y^{\prime}\right)$ and $\left(f^{\prime}, g^{\prime}\right):\left(e^{\prime}, \mathcal{B}^{X^{\prime}}, Y^{\prime}\right) \rightarrow\left(e^{\prime \prime}, \mathcal{B}^{X^{\prime \prime}}, Y^{\prime \prime}\right)$ are TEXT-morphisms, then 
they can be composed according to the rule:

$$
\left(f^{\prime}, g^{\prime}\right) \circ(f, g):=\left(f^{\prime} \circ f, g^{\prime} \circ g\right):\left(e, \mathcal{B}^{X}, X\right) \rightarrow\left(e^{\prime \prime}, \mathcal{B}^{X^{\prime \prime}}, Y^{\prime \prime}\right)
$$

where "。” denotes the composition of maps.

Remark 1.6 Observe, that axiom $\left(t x_{1}\right)$ in this definition is automatically satisfied if $e: X \rightarrow Y$ is a topological embedding. Moreover we admit an ordinary $\underline{B}$-set $\mathcal{B}^{X}$ on $X$ which need not be necessary coincide with the power $\underline{P} X$. In addition we mention that such an extension is called

(1) strict iff $\left\{c l_{Y}(e[A]): A \subset X\right\}$ forms a base for the closed subsets of $Y$ [7];

(2) symmetric iff $x \in X$ and $y \in c l_{Y}(\{e(x)\})$ imply $e(x) \in c_{Y}(\{y\})$ [8].

Examples 1.7 (i) For a topological extension $\left(e, \mathcal{B}^{X}, Y\right)$ we consider the triple $\left(X, \mathcal{B}^{X}, N_{e}\right)$, where

$$
N_{e}(B):=\left\{\rho \subset \underline{P} X: \exists y \in e[B] y \in \bigcap\left\{c l_{Y}(e[A]): A \in \rho\right\}\right\}
$$

if $B \neq \varnothing$ and $N_{e}(\varnothing):=\{\varnothing\}$;

(ii) For a symmetric topological extension $\left(e, \mathcal{B}^{X}, Y\right)$ we consider the triple $\left(X, \mathcal{B}^{X}, N^{e}\right)$, where

$$
N^{e}(B):=\left\{\rho \subset \underline{P} X: \exists y \in c l_{Y}(e[B]) y \in\left\{c l_{Y}(e[A]): A \in \rho\right\}\right\}
$$

if $B \neq \varnothing$ and $N^{e}(\varnothing):=\{\varnothing\}$.

\section{Fundamental Classes of Prehypernear Spaces}

With respect to above examples, first let us focus our attention to some important classes of prehypernear spaces.

Definitions 2.1 A prehypernear space $\left(X, \mathcal{B}^{X}, N\right)$ is called

(i) saturated iff $X \in \mathcal{B}^{X}$;

(ii) discrete iff $\mathcal{B}^{X}:=\{\varnothing\} \cup\{\{x\}: x \in X\}$;

(iii) symmetric iff $B \in \mathcal{B}^{X} \backslash\{\varnothing\}$ and $\rho \in N(B)$ imply $\{B\} \cup \rho \in N(B)$ and $\{B\} \cup \rho \in \cap\left\{N(F): F \in \rho \cap \mathcal{B}^{X}\right\}$;

(iv) pointed iff $B \in \mathcal{B}^{X} \backslash\{\varnothing\}$ implies $N(B)=\cup\{N(\{x\}): x \in B\}$;

(v) conic iff $B \in \mathcal{B}^{X}$ implies $\cup\{\rho \subset \underline{P} X: \rho \in N(B)\} \in N(B)$;

(vi) set-defined iff $B \in \mathcal{B}^{X}$ implies $\{A \subset X: B \cap A \neq \varnothing\}=: \breve{B} \in N(B)$.

Theorem 2.2 The category PNEAR of prenearness spaces and related maps is isomorphic to the category

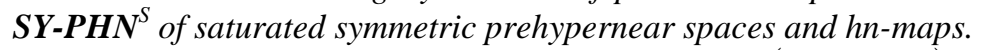

Proof. According to Example 1.3. (i) we claim that $\left(X, \underline{P} X, N_{\xi}\right)$ is a symmetric saturated prehypernear space. Conversely, we consider for such proposed space $\left(Y, \mathcal{B}^{\bar{Y}}, M\right)$ the following prenearness space $\left(Y, \eta_{M}\right)$ defined by setting:

$$
\eta_{M}:=\{\mathcal{A} \subset \underline{P} X: \mathcal{A} \in \bigcap\{M(A): A \in \mathcal{A}\}\} .
$$

Hence, the above mentioned connections are functoriell, and thus it remains to prove that the following two statements are valid, i.e.

(i) $\xi=\eta_{N_{\xi}}$;

(ii) $M=N_{\eta_{M}}$.

To (i): " $\subset$ "; $\mathcal{A} \in \xi$ and $A \in \mathcal{A}$ imply $A \neq \varnothing$, hence $\{A\} \cup \mathcal{A} \in \xi$, and $\mathcal{A} \in N_{\xi}(A)$ is valid which shows $\mathcal{A} \in \eta N_{\xi}$.

“ $\supset$ ”: $\mathcal{A} \in \eta_{N_{\xi}}$ and without restriction $A \neq \varnothing$. Choose $\varnothing \neq A \in \mathcal{A}$, hence $\mathcal{A} \in N_{\xi}(A)$ by hypothesis, and $\{A\} \cup \mathcal{A} \in \xi$ follows.

Since $\mathcal{A} \ll\{A\} \cup \mathcal{A}$ we claim $\mathcal{A} \in \xi$.

To (ii): “ $\leq$ ”: Without restriction let be $\varnothing \neq A \in \underline{P} X$.

For $\mathcal{A} \in M(A)$ we have to verify $\{A\} \cup \mathcal{A} \in \eta_{M}$. So, let be $A^{\prime} \in \mathcal{A}$, hence $\{A\} \cup \mathcal{A} \in M\left(A^{\prime}\right)$ since $M$ 
is symmetric and saturated by hypothesis. Consequently, $\mathcal{A} \in N_{\eta_{M}}(A)$ is valid.

" $\geq$ " Conversely, let be $\mathcal{A} \in N_{\eta_{M}}(A)$, hence $\{A\} \cup \mathcal{A} \in \eta_{M}$. Choose $\varnothing \neq A^{\prime} \in \mathcal{A}$ (according to $\left(\mathrm{hn}_{1}\right)$ respectively $\left.\left(\mathrm{hn}_{3}\right)\right)$. Thus $\{A\} \cup \mathcal{A} \in M\left(A^{\prime}\right)$ holds, and $\left\{A^{\prime}\right\} \cup(\{A\} \cup \mathcal{A}) \in M(A)$ follows by hypothesis. But $\mathcal{A} \ll\left\{A^{\prime}\right\} \cup(\{A\} \cup \mathcal{A})$, hence $\mathcal{A} \in M(A)$ is valid.

Remark 2.3 In this context we point out that each prehypernear space $\left(X, \mathcal{B}^{X}, N\right)$ induces in general the following $\breve{C}$ ech-closure operators by setting:

(1) $\operatorname{cl}_{N}(A):=\{x \in X:\{A\} \in N(\{x\})\}$;

(2) $\operatorname{cl}^{N}(A):=\{x \in X:\{\{x\}, A\} \in N(\{x\})\}$,

where the following inclusion is valid: $A \in \underline{P} X$ implies $\mathrm{cl}^{N}(A) \subset c l_{N}(A)$. In the symmetric case these two operators coincide, moreover we have $x \in C l_{\xi}(A)$ iff $x \in C l_{N_{\xi}}(A)$, and finally $\left(X, c l_{N}\right)$ defines a symmetric C ech-closure space.

Definition 2.4 A prehypernear space $\left(X, \mathcal{B}^{X}, N\right)$ is called a pseudohypernear space iff $N$ is isoton, i.e. $N$ satisfies (is) $B_{1} \subset B_{2} \in \mathcal{B}^{X}$ imply $N\left(B_{1}\right) \subset N\left(B_{2}\right)$. We denote by PSHN the corresponding full subcategory of $\mathrm{PHN}$.

Remark 2.5 In this context we refer to Examples 1.3. (i), (iv), (v), (vi), (vii), respectively Examples 1.7. (i), (ii).

Theorem 2.6 The category Č-CLO of Čech-closure spaces and continuous maps is isomorphic to a full subcategory of PSHN.

Remark 2.7 Now, before showing the above mentioned theorem we give the following definition.

Definition 2.8 A prehypernear space $\left(X, \mathcal{B}^{X}, N\right)$ is called sected iff $N$ satisfies (sec), i.e.

(sec) $B \in \mathcal{B}^{X}$ and $\rho \in N(B)$ imply $B \in \sec \left\{c l_{N}(F): F \in \rho\right\}$.

Remark 2.9 In this connexion we point out that each pointed prehypernear space (see Remark 3.6) is always sected.

Moreover, sected prehypernear spaces are already pseudohypernear spaces.

Definition 2.10 A sected conic saturated prehypernear space is called closed, and we denote by CL-PHSN the full subcategory of PSHN, whose objects are closed pseudohypernear spaces.

Proof of Theorem 2.6.

According to Example 1.3. (v) we claim that $\left(X, \underline{P} X, N^{-}\right)$is a closed pseudohypernear space. Conversely, we consider for such proposed space $\left(Y, \mathcal{B}^{Y}, M\right)$ the Cech-closure space $\left(Y, c l_{M}\right)$. Hence, the above mentioned connections are functoriell, and thus it remains to prove that the following two statements are valid, i.e.:

(i) $-=\mathrm{Cl}_{N^{-}}$;

(ii) $M=N^{c l_{M}}$.

To (i): Now let be $A \in \underline{P} X$, we have to verify $\bar{A}=c l_{N^{-}}(A)$. Firstly, $x \in \bar{A}$ implies $\{x\} \in \sec \{\bar{A}\}$, hence $\{A\} \in N^{-}(\{x\})$, and $x \in c \bar{l}_{N^{-}}(A)$ results.

Secondly, $x \in c_{N^{-}}(A)$ implies $\{A\} \in N^{-}(\{x\})$, hence $\{x\} \in \sec \{\bar{A}\}$, and $x \in \bar{A}$ follows.

To (ii): Now, let be without restriction $A \in \underline{P} X \backslash\{\varnothing\} . \rho \in M(A)$ and $\mathcal{F} \in \rho$ imply $\{F\} \in M(A)$ according to $\left(\mathrm{hn}_{1}\right)$, hence $A \cap c l_{M}(F) \neq \varnothing$ by hypothesis, and $\rho \in N^{c_{M}}(A)$ results.

Conversely, $\rho \in N^{c_{M}}(A)$ implies $A \in \sec \left\{c l_{M}(F): F \in \rho\right\}$.

Now, we will show that $\rho \subset \bigcup\{\mathcal{A} \subset \underline{P} X: \mathcal{A} \in M(A)\} . F \in \rho$ implies $A \cap \operatorname{cl}_{M}(F) \neq \varnothing$ by hypothesis. Choose $x \in A$ with $\{F\} \in M(\{x\})$, hence $\{F\} \in M(A)$, since $M$ satisfies (is). But then

$$
\rho \subset \bigcup\{\mathcal{A} \subset \underline{P} X: \mathcal{A} \in M(A)\} \in M(A)
$$

is valid which implies $\rho \in M(A)$, hence concluding the proof.

Remark 2.11 Now, in the following another important class of prehypernear spaces will be examined, being fruitful in considering convergence problems and having those properties, which are characterizing topological universes.

\section{Grill-Spaces}

Definitions 3.1 A prehypernear space $\left(X, \mathcal{B}^{X}, N\right)$ is called a prehypergrill space iff $\mathrm{N}$ satisfies (gri), i.e. 
(gri) $B \in \mathcal{B}^{X}$ and $\rho \in N(B)$ imply there exists $\gamma \in \operatorname{GRL}(X) \rho \subset \gamma \in N(B)$, where $\operatorname{GRL}(X):=\{\gamma \subset \underline{P} X: \gamma$ is grill $\}$, and $\gamma \subset \underline{P} X$ is called grill (Choquet [9]) iff it satisfies

( (gii $\left._{1}\right) \varnothing \notin \gamma$;

$\left(\mathrm{gri}_{2}\right) G_{1} \cup G_{2} \in \gamma$ iff $G_{1} \in \gamma$ or $G_{2} \in \gamma$.

We denote by $\mathbf{G}-\mathbf{P H N}$ the category, whose objects are the prehypergrill spaces with hn-maps between them and by $\mathbf{G}-\mathbf{P H} \boldsymbol{N}^{\bullet}$ the category, whose objects are the prehypergrill spaces with shn-maps between them.

Remark 3.2 We refer to Examples 1.3. (ii), (iii), (iv), (vi), (vii) respectively and to Examples 1.7. (i), (ii).

Theorem 3.3 The category GRILL of grill-determined prenearness spaces and nearness preserving maps is isomorphic to a full subcategory of $\mathbf{G}-\mathbf{P H N}$.

Proof. According to Theorem 2.2 we already know that $\left(X, \underline{P} X, N_{\xi}\right)$ is a symmetric saturated prehypernear space, hence additionally it is a prehypergrill space by hypothesis. Conversely, $\eta_{M}$ is grill-determined by supposition.

Theorem 3.4 The category SETCONV ([3]) of set-convergence spaces and related maps is isomorphic to a full subcategory of $\mathbf{G}-\mathbf{P H N}^{\bullet}$.

Proof. According to Example 1.3. (iii) we claim that the triple $\left(X, \mathcal{M}^{X}, N_{q}\right)$ is a set-defined prehypergrill space. Conversely, we consider for such proposed space $\left(Y, \mathcal{B}^{Y}, M\right)$ the following set-convergence space $\left(Y, \mathcal{B}^{Y}, p_{M}\right)$ defined by setting: $\mathcal{F}_{p_{M}} B$ iff $\sec \mathcal{F} \in M(B)$ for each $B \in \mathcal{B}^{X}$. Hence, the above mentioned connections are functoriell with respect to shn-maps. Thus, it remains to prove that the following two statements are valid, i.e.

(i) $q=p_{N_{a}}$;

(ii) $M=N_{p_{M}}$.

To (i) " $\leq$ ” $\mathcal{F} q B$ implies $\mathcal{F} p_{N_{q}} B$, evidently.

" $\geq$ ”: $\mathcal{F} p_{N_{q}} B$ implies $\sec \mathcal{F} \in N_{q}(B)$, hence there exists $\mathcal{F}^{\prime} \in \operatorname{FIL}(X) \sec \mathcal{F} \subset \sec \mathcal{F}^{\prime}$ and $\mathcal{F}^{\prime} q B$. Since $\mathcal{F}^{\prime} \subset \mathcal{F}$ we conclude with $\mathcal{F} q B$.

To (ii): “ $\leq$ ” $\rho \in M(B)$ implies the existence of $\gamma \in \operatorname{GRL}(X)$ with $\rho \subset \gamma \in M(B)$. Consequently, $\sec \gamma \in \operatorname{FIL}(X)$ and $\sec \gamma p_{M} B$ results, hence $\rho \in N_{p_{M}}(B)$ is valid.

" $\geq ": \rho \in N_{p_{M}}(B)$ implies that $\rho \subset \sec \mathcal{F}$ and $\mathcal{F}_{p_{M}} B$ hold. Hence $\sec \mathcal{F} \in M(B)$, and $\rho \in M(B)$ results.

Corollary 3.5 The category GCONV of generalized convergence spaces and related maps is isomorphic to the category DISG-PHN ${ }^{*}$, whose objects are the discrete prehypergrill soaces and whose morphisms are the sected hn-maps.

Remark 3.6 Now, in this connextion it is interesting to note that there exists and alternate description of generalized convergence spaces in the realm of prehypergrill spaces. Analogously, how to describing set convergence on arbitrary B-sets we offer now a corresponding one for the point convergence as follows: Let be given a point-convergence space $(X, q)$, where $q \subset \operatorname{FIL}(X) \times X$ is satisfying some natural conditions. Then we consider the following pointed prehyergrill space $\left(X, \underline{P} X, N^{q}\right)$ by setting $N^{q}(\varnothing):=\{\varnothing\}$ and

$$
N^{q}(B):=\{\rho \subset \underline{P} X: \exists x \in B \exists \mathcal{F} \in \operatorname{FIL}(X)(\rho \subset \sec \mathcal{F} \text { and } \mathcal{F} q x)\}
$$

if $B \neq \varnothing$.

Conversely let be given a pointed saturated prehypergrill space $\left(Y, \mathcal{B}^{Y}, M\right)$ then we naturally define a pointconvergence space $\left(Y, p_{M}\right)$ by setting $\mathcal{F}_{p_{M}} y$ iff $\sec \mathcal{F} \in M(\{y\})$. As a consequence we obtain the result that point convergence can be essentially expressed by means of its corresponding pointed saturated prehypergrill spaces and sected hn-maps.

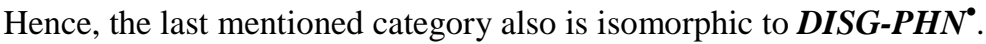

Remark 3.7 Another interesting fact is the following one. As Wyler has shown in [3] supertopological spaces in the sense of Doîtchînov can be regarded as special set-convergence spaces. Hence it is also possible for describing them in the realm of prehypergrill spaces. Concretely let be given a supertopological space (see [10]) or more generally a neighborhood space $\left(X, \mathcal{M}^{X}, \Theta\right)$ in the sense of [6], in the following referred as to presupertopological space. Then we consider the triple $\left(X, \mathcal{M}^{X}, N_{\Theta}\right)$, where $N_{\Theta}(B):=\{\rho \subset \underline{P} X: \rho \subset \sec \Theta(B)\}$ for each $\mathcal{B} \in \mathcal{M}^{X}$. Hence the triple $\left(X, \mathcal{M}^{X}, N_{\Theta}\right)$ is a conic pseudohypergrill space. Hereby, a prehypergrill space $\left(X, \mathcal{B}^{X}, N\right)$ is called pseudohypergrill space iff $N$ satisfies (is) (see also Definition 2.4). By CG-PSHN respectively $\mathbf{C G}-\mathbf{P S H} \mathbf{N}^{*}$ we denote the corresponding categories. At last we point out that conic pseudohyper- 
near spaces are even set-defined.

Theorem 3.8 The category PRESTOP of presupertopological spaces and continuous maps is isomorphic to the category $\mathbf{C G}-\mathbf{P S H} \mathbf{N}^{\bullet}$.

Proof. According to Remark 3.7 we consider conversely for a conic pseudohypergrill space $\left(Y, \mathcal{B}^{Y}, M\right)$ the space $\left(Y, \mathcal{B}^{Y}, \odot_{M}\right)$, where for each $B \in \mathcal{B}^{Y} \odot_{M}$ is defined by setting:

$$
\odot_{M}(B):=\sec \bigcup\{\gamma \in \operatorname{GRL}(Y): \gamma \in M(B)\}
$$

for each $B \in \mathcal{B}^{Y}$. Then $\left(Y, \mathcal{B}^{Y}, \odot_{M}\right)$ is a presupertopological space. Hence, the above mentioned connections are functoriell with respect to shn-maps. Thus, it remains to prove that the following two statements are valid, i.e.

(i) $\Theta={ }^{\odot} N_{\Theta}$;

(ii) $M={ }^{N} \bigodot_{M}$.

To (i): " $\leq$ ": For $B \in \mathcal{M}^{X}$ let be $U \in \Theta(B) . F \in \cup\left\{\gamma \in \operatorname{GRL}(X): \gamma \in N_{\Theta}(B)\right\}$ implies the existence of $\gamma \in N_{\Theta}(B)$ with $F \in \gamma$, hence $\gamma \subset \sec \Theta(B)$ follows. Consequently $F \in \sec \Theta(B)$ is valid, showing that $F \cap U \neq \varnothing$.

“ $\geq$ ": Since $\sec \Theta(B) \in N_{\Theta}(B)$ is grill we get $\sec \Theta(B) \subset \bigcup\left\{\gamma \in \operatorname{GRL}(X): \gamma \in N_{\Theta}(B)\right\}$, hence

$$
\sec \bigcup\left\{\gamma \in \operatorname{GRL}(X): \gamma \in N_{\Theta}(B)\right\} \subset \Theta(B)
$$

is valid.

To (ii): “ $\leq$ ” $\rho \in M(B)$ implies the existence of $\gamma \in \mathrm{GRL}(X)$ with $\rho \subset \gamma \in M(B)$ by hypothesis, hence $\rho \subset \sec \Theta_{M}(B)$.

“ $\geq " \quad \rho \in N_{\odot_{M}}(B)$ implies $\rho \subset \sec \Theta_{M}(B)=\bigcup\{\gamma \in \operatorname{GRL}(X): \gamma \in M(B)\} \in M(B)$, hence $\rho \in M(B)$ according to $\left(\mathrm{hn}_{1}\right)$.

Remark 3.9 $b$-proximities (see [6]) are of significant importance when considering topological extensions. Here we will give two interesting examples in that direction as follows:

(1) For a symmetric topological space $(Y, t)$ (given by a closure operator $t$ ) let $\mathcal{B}^{X}$ be a $\underline{B}$-set with $X \subset Y$, then we define a $b$-proximity $\delta_{t} \subset \mathcal{B}^{X} \times \underline{P} X$ by setting: $B \delta_{t} A$ iff $t(B) \cap t(A) \neq \varnothing$ for each $B \in \mathcal{B}^{X}$ and $A \subset X$. Now, it is easy to verify that $\delta_{t}$ is $t$-compatible, which means the equality $c l_{\delta_{t}}=t$ holds by restricting $t$ on $X$, where $c l_{\delta_{t}}$ denotes the closure-operator induced by $\delta_{t}$.

(2) Let being the same hypothesis as in (1). We set $\mathcal{B}_{t}^{X}:=\left\{D \subset X: \exists B \in \mathcal{B}^{X} t(B) \supset D\right\}$ and define a nearness relation $\delta^{t} \subset \mathcal{B}_{t}^{X} \times \underline{P} X$ by setting: $D \delta^{t} A$ iff $t(D) \cap t(A) \neq \varnothing$. Then $\delta^{t}$ defines a $b$-proximity with the same properties as mentioned above. Now, we recall the definition of a $b$-proximity respectively $b$-proximity space as follows:

Definition 3.10 A $b$-proximity space consists of a triple $\left(X, \mathcal{B}^{X}, \delta\right)$, where $X$ is set, $\mathcal{B}^{X} \underline{B}$-set and $\delta \subset \mathcal{B}^{X} \times \underline{P} X \quad$ satisfying the following conditions:

$\left(\mathrm{bp}_{1}\right) \varnothing \bar{\delta} A$ and $B \bar{\delta} \varnothing$ (i.e. $\varnothing$ is not in relation to $A$, and analogously this is also holding for $B$ );

$\left(\mathrm{bp}_{2}\right) \quad B \delta\left(A_{1} \cup A_{2}\right)$ iff $B \delta A_{1}$ or $B \delta A_{2}$;

$\left(\mathrm{bp}_{3}\right) \quad x \in X$ implies $\{x\} \delta\{x\}$;

(bp $\mathrm{b}_{4} B_{1} \subset B_{2} \in \mathcal{B}^{X}$ and $B_{1} \delta A$ imply $B_{2} \delta A$.

Remark 3.11 Here we point out that b-proximities are in one-to-one correspondence with presupertopologies. In the symmetric case, if $\delta$ additionally satisfies (sbp), i.e.

(sbp) $B_{1}, B_{2} \in \mathcal{B}^{X}$ and $B_{1} \delta B_{2}$ imply $B_{2} \delta B_{1}$ and moreover $\mathcal{B}^{X}$ equals $\underline{P} X$, then symmetric $b$-proximities coincide with the Čech-proximities mentioned by Deák ([11]).

Definition 3.12 For $b$-proximity spaces $\left(X, \mathcal{B}^{X}, \delta\right),\left(Y, \mathcal{B}^{Y}, \gamma\right)$ a bounded function $f: X \rightarrow Y$ is called p-map iff $f$ satisfies (p), i.e.

(p) $B \in \mathcal{B}^{X}, A \in \underline{P} X$ and $B \delta A$ imply $f[B] \gamma f[A]$. By $\boldsymbol{b}$-PROX we denote the corresponding category.

Theorem 3.13 The category b-PROX and CG-PSHN are isomorphic.

Proof. For a $b$-proximity space $\left(X, \mathcal{M}^{X}, \delta\right)$ we consider the triple $\left(X, \mathcal{M}^{X}, N_{\delta}\right)$, where

$$
N_{\delta}(B):=\{\rho \subset \underline{P} X: \rho \subset \delta(B)\}
$$


for each $B \in \mathcal{M}^{X}$ with $\delta(B):=\{A \subset X: B \delta A\}$. Then $\left(X, \mathcal{M}^{X}, N_{\delta}\right)$ is a conic pseudohypergrill space. Conversely let be given such a space $\left(Y, \mathcal{B}^{Y}, M\right)$, then we consider the triple $\left(Y, \mathcal{B}^{Y}, p_{M}\right)$, where $p_{M} \subset \mathcal{B}^{Y} \times \underline{P} Y$ is defined by setting $B_{p_{M}} A$ iff $\{A\} \in M(B)$ for each $B \in \mathcal{B}^{Y}$ and $A \subset Y$. Hence, $\left(Y, \mathcal{B}^{Y}, p_{M}\right)$ is a b-proximity space. The above mentioned connections are functoriell, and thus it remains to prove that the following two statements are valid, i.e.

(i) $\delta=p_{N_{\delta}}$;

(ii) $M=N_{p_{M}}$.

To (i): “ $\subset$ ”: $B \delta A$ implies $\{A\} \in N_{\delta}(B)$, hence $B p N_{\delta} A$.

“ $\supset$ ”: $B p_{N_{\delta}} A$ implies $\{A\} \in N_{\delta}(B)$, hence $B \delta A$ follows.

To (ii): " $\leq$ ": $\rho \in M(B)$ and $F \in \rho$ imply $\{F\} \in M(B)$, hence $B p_{M} F$, and $F \in p_{M}(B)$ results.

“ $\geq$ ” $\rho \in N_{p_{M}}(B)$ implies $\rho \subset p_{M}(B)$. We will show that $\rho \subset \bigcup\{\mathcal{A} \subset \underline{P} X: \mathcal{A} \in M(B)\}$.

$F \in \rho$ implies $F \in p_{M}(B)$ by hypothesis, hence $B p_{M} F$, and $\{F\} \in M(B)$ results which concludes the proof.

Résumé 3.14 Respecting to former advisements we note that we have established only some topological concept in which some important classical ones can be now expressed and studied in a very natural way. Moreover, the fundamental categories how as GRILL, b-PROX, PRESTOP, GCONV and SETCONV can be regarded as special subcategories of $\mathbf{G - P H N}$. (see also the Theorem 3.3, 3.4, 3.8 and 3.13 respectively).

\section{Bonding in Prehypernear Spaces}

A slight modification of the definition for being a prehypergrill space leads us to the following notation.

Definition 4.1 A prehypernear space $\left(X, \mathcal{B}^{X}, N\right)$ is called bonded iff $N$ satisfies (b), i.e.

(b) $B \in \mathcal{B}^{X}$ and $\rho_{1} \vee \rho_{2} \in N(B)$ imply $\rho_{1} \in N(B)$ or $\rho_{2} \in N(B)$, where $\rho_{1} \vee \rho_{2}:=\left\{F_{1} \cup F_{2}: F_{1} \in \rho_{1}, F_{2} \in \rho_{2}\right\}$.

Remark 4.2 Each prehypergrill space is bonded.

Proof. evident.

Definition 4.3 Now, we call a bonded pseudohypernear space a semihypernear space and denote by $\boldsymbol{S H N}$ the full subcategory of $\boldsymbol{P S H N}$.

Theorem 4.4 The category PrTOP of pretopological spaces and continuous maps is isomorphic to a full subcategory of $\mathrm{SHN}$.

Proof. According to Theorem 2.6 respectively Definition 2.10 it is evident that $c l_{M}$ additionally satisfies $c l_{M}\left(A_{1} \cup A_{2}\right)=c l_{M}\left(A_{1}\right) \cup c l_{M}\left(A_{2}\right)$, hence being a pretopology on its underlying set. On the other hand $N^{-}$is bonded, because $\rho_{1} \vee \rho_{2} \in N^{-}(B)$ implies $B \in \sec \left\{\bar{F}: F \in \rho_{1} \vee \rho_{2}\right\}$. We suppose that $\rho_{1}, \rho_{2} \notin N^{-}(B)$, hence there exist $F_{1} \in \rho_{1}, \quad F_{2} \in \rho_{2}$ such that $B \cap \bar{F}_{1}=\varnothing=B \cap \bar{F}_{2}$. Consequently,

$$
\varnothing=\left(B \cap \bar{F}_{1}\right) \cup\left(B \cap \bar{F}_{2}\right)=B \cap\left(\bar{F}_{1} \cup \bar{F}_{2}\right)=B \cap \overline{F_{1} \cup F_{2}} .
$$

But $F_{1} \cup F_{2} \in \rho_{1} \cup \rho_{2}$ leads us to a contradiction.

Theorem 4.5 The category SNEAR of seminearness spaces and related maps is isomorphic to a full subcategory of SHN.

Proof. According to Theorem 2.2 we firstly show that $N_{\xi}$ is bonded. Without restriction bet be $B \in \mathcal{B}^{X} \backslash(\varnothing)$ and $\rho_{1} \vee \rho_{2} \in N_{\xi}(B)$, hence $\{B\} \cup\left(\rho_{1} \vee \rho_{2}\right) \in \xi$. Since $\left(\{B\} \cup \rho_{1}\right) \vee\left(\{B\} \cup \rho_{2}\right) \ll\{B\} \cup\left(\rho_{1} \vee \rho_{2}\right)$ we obtain $\left(\{B\} \cup \rho_{1}\right) \vee\left(\{B\} \cup \rho_{2}\right) \in \xi$. Thus $\{B\} \cup \rho_{1} \in \xi$ or $\{B\} \cup \rho_{2} \in \xi$ results, showing that $N_{\xi}$ is satisfying (b). On the other hand let be $\mathcal{A}_{1} \vee \mathcal{A}_{2} \in \eta_{M}$ and without restriction $\mathcal{A}_{1} \neq \varnothing \neq \mathcal{A}_{2}$. We suppose $\mathcal{A}_{1}, \mathcal{A}_{2} \notin \eta_{M}$, hence there exist $A_{1} \in \mathcal{A}, A_{2} \in \mathcal{A}_{2}$ with $\mathcal{A}_{1} \notin M\left(A_{1}\right)$ and $\mathcal{A}_{2} \notin M\left(A_{2}\right)$. Consequently $A_{1} \cup A_{2} \in \mathcal{A}_{1} \vee \mathcal{A}_{2}$ follows. Then we get $\mathcal{A}_{1} \vee \mathcal{A}_{2} \in M\left(A_{1} \cup A_{2}\right)$ by hypothesis. Since $M$ is bounded we have $\mathcal{A}_{1} \in M\left(A_{1} \cup A_{2}\right)$, or $\mathcal{A}_{2} \in M\left(A_{1} \cup A_{2}\right)$. By symmetry of $M$ we obtain the statement $\left\{A_{1} \cup A_{2}\right\} \cup \mathcal{A}_{1} \in M\left(A_{1}\right)$ or $\left\{A_{1} \cup A_{2}\right\} \cup \mathcal{A}_{2} \in M\left(A_{2}\right)$. Consequently, $\mathcal{A}_{1} \in M\left(A_{1}\right)$ or $\mathcal{A}_{2} \in M\left(A_{2}\right)$ leads us to a contradiction.

Remark 4.6 A pseudohypernear space $\left(X, \mathcal{B}^{X}, N\right)$ induces two underlying psb-hull operators by setting for each $B \in \mathcal{B}^{X}$ : 


$$
\begin{aligned}
& \bar{B}^{N}:=\{x \in X:\{\{x\}\} \in N(B)\} ; \\
& { }^{N} \bar{B}:=\{x \in X:\{\{x\}, B\} \in N(B)\},
\end{aligned}
$$

whereby the inclusion ${ }^{N} \bar{B} \subset \bar{B}^{N}$ is valid for each $B \in \mathcal{B}^{X}$. If $N$ is symmetric then the two operators coincide, and moreover we claim the following equalities for each $B \in \mathcal{B}^{X}$, i.e. $c l_{N}(B)=c l^{N}(B)={ }^{N} \bar{B}=\bar{B}^{N}$. Hereby, a function $h: \mathcal{B}^{X} \rightarrow \underline{P} X$ is called a psb-hull operator, and the triple $\left(X, \mathcal{B}^{X}, h\right)$ is called a psb-hull space iff $h$ satisfies the following conditions:

$\left(\mathrm{bh}_{1}\right) h(\varnothing)=\varnothing$;

$\left(\mathrm{bh}_{2}\right) \quad B \in \mathcal{B}^{X}$ implies $B \subset h(B)$;

$\left(\mathrm{bh}_{3}\right) \quad B_{1} \subset B_{2} \in \mathcal{B}^{X}$ imply $h\left(B_{1}\right) \subset h\left(B_{2}\right)$.

For psb-hull spaces $\left(X, \mathcal{B}^{X}, h_{X}\right),\left(Y, \mathcal{B}^{Y}, h_{Y}\right)$ let $f: X \rightarrow Y$ be a bounded function, then $f$ is called $b$ continuous iff $B \in \mathcal{B}^{X}$ implies $f\left[h_{X}(B)\right] \subset h_{Y}(f[B])$. We denote by Psb-HULL the corresponding category.

Definition 4.7 Now, we call a conic pseudohypernear space $\left(X, \mathcal{B}^{X}, N\right)$ a pseudohull space iff $N$ satisfies (h), i.e.

(h) $B \in \mathcal{B}^{X}$ and $\rho \in N(B)$ imply $\bar{B}^{N} \in \sec \rho$. We denote by PSHU the full subcategory of PSHN, whose objects are the pseudohull spaces.

Theorem 4.8 The categories Psb-HULL and PSHU are isomorphic.

Proof. According to Remark 4.6 we already know that $\left(X, \mathcal{B}^{X},-N\right)$ is a psb-hull space. Conversely, for a psb-hull space $\left(Y, \mathcal{B}^{Y}, h\right)$ we consider the triple $\left(Y, \mathcal{B}^{Y}, M^{h}\right)$ by setting for each

$$
\mathcal{B} \in \mathcal{B}^{Y}: M^{h}(B):=\{\rho \subset \underline{P} X: h(B) \in \sec \rho\} .
$$

Then $\left(Y, \mathcal{B}^{Y}, M^{h}\right)$ is a pseudohull space. Hence, the above mentioned connections are functoriell. Thus it remains to prove that the following two statements are valid, i.e.

(i) $h=-M^{h}$;

(ii) $N=M^{-N}$.

To (i): " $\leq$ ”: For $B \in \mathcal{B}^{X}$ let be $x \in h(B)$, hence $h(B) \in \sec \{\{x\}\}$. Consequently, $\{\{x\}\} \in M^{h}(B)$ follows, showing that $x \in \bar{B}^{M^{h}}$ is valid.

“ $\geq$ ”: evident.

To (ii): " $\leq$ ": $\rho \in N(B)$ and $F \in \rho$ imply $\bar{B}^{N} \cap F \neq \varnothing$ by hypothesis, hence $\rho \in M^{-N}(B)$ is valid.

" $\geq$ ": $\rho \in M^{-N}(B)$; we will show that $\rho \subset \cup\{F \subset X: \exists \mathcal{A} \in N(B) F \in \mathcal{A}\}$ is valid. $F \in \rho$ implies $\bar{B}^{N} \cap F \neq \varnothing$ by hypothesis. Choose $x \in F$ with $\{\{x\}\} \in N(B)$, hence $\{F\} \in N(B)$ according to (hn ${ }_{1}$ ). Consequently, the above mentioned inclusion is valid, showing that $\rho \in N(B)$.

Corollary 4.9 In the saturated case CL-PSHN and PSHU are isomorphic categories.

Proof. We refer to Theorem 2.6, Definition 2.10 and Theorem 4.8 respectively.

Definition 4.10 A prehypernear space $\left(X, \mathcal{B}^{X}, N\right)$ is called connected if $N$ satisfies (cnc), i.e.

(cnc) $B_{1}, B_{2} \subset X$ and $B_{1} \cup B_{2} \in \mathcal{B}^{X}$ imply $N\left(B_{1} \cup B_{2}\right)=N\left(B_{1}\right) \cup N\left(B_{2}\right)$.

Remark 4.11 We note that each pointed prehypernear space is connected, moreover this also is holding for any symmetric semihypernear space. Consequently, the underlying psb-hull operator $-N$ additionally satisfying (ad), i.e.

(ad) $h\left(B_{1} \cup B_{2}\right)=h\left(B_{1}\right) \cup h\left(B_{2}\right)$. Now, let us call such an operator $h$-hull operator, and we denote by $\boldsymbol{b}$-HULL the corresponding full subcategory of Psb-HULL with related objects. In the saturated case we claim that $\boldsymbol{b}-\boldsymbol{H} \boldsymbol{U} \boldsymbol{L} \boldsymbol{L}$ and $\boldsymbol{C L}-\boldsymbol{S H N}$ are isomorphic categories. Hereby $\boldsymbol{C L}$-SHN denotes the full subcategory of $\boldsymbol{S H N}$, whose objects are the closed semihypernear spaces.

\section{Hypernear Spaces}

As already observed, hypertopologies appear in connexion with certain interior operators studied by Kent and Min ([12]). Hereby a function - $\underline{P} X \rightarrow \underline{P} X$ is called a hypertopology on $X$, and the pair $(X,-)$ is called a hypertopological space iff “-” satisfies the following conditions: 
(hyt $\left.{ }_{1}\right) \bar{\varnothing}=\varnothing$;

(hyt 2 ) $A \in \underline{P} X$ implies $A \subset \bar{A}$;

(hyt $\left.{ }_{3}\right) \quad A_{1} \subset A_{2} \in \underline{P} X \quad$ imply $\bar{A}_{1} \subset \bar{A}_{2}$;

(hyt $\left.{ }_{4}\right) \quad A \in \underline{P} X$ implies $\overline{\bar{A}} \subset \bar{A}$.

For hypertopological spaces $(X,-X),(Y,-Y)$ let $f: X \rightarrow Y$ be a function, then $f$ is called continuous iff $A \in \underline{P} X$ implies $\left.f\left[\bar{A}^{X}\right] \subset \overline{f[A]}\right]^{Y}$. By HYTOP we denote the corresponding subcategory of $\breve{C}-C L O$. Evidenly, the category TOP of topological spaces and continuous maps can be now regarded as a special case of HYTOP. On the other hand certain nearnesses play an important role in the realm of unifications and extensions, respectively. This is holding for distinguished nearness spaces and $b$-proximity spaces in fact. Moreover, certain supertopologies are involved, too. Now, in the following we will give a common description of them all by introducing the so called concept of a hypernear space.

Definition 5.1 A pseudohypernear space $\left(X, \mathcal{B}^{X}, N\right)$ is called a hypernear space iff $N$ satisfies (hn), i.e.

(hn) $B \in \mathcal{B}^{X}$ and $\left\{c l_{N}(F): F \in \rho\right\} \in N(B)$ imply $\rho \in N(B)$.

We denote by $\boldsymbol{H N}$ the corresponding full subcategory of $\boldsymbol{P S H N}$. Note, that in this case $c l_{N}$ is a hypertopology on $X$.

Theorem 5.2 CL-HN denotes the full subcategory of CL-PSHN, whose objects are the closed hypernear spaces, then $\mathbf{C L}-\mathbf{H N}$ and HYTOP are isomorphic.

Proof. The reader is referred to Theorem 2.6 and Definition 2.10, respectively.

Remark 5.3 As pointed out in Remark 3.6, point convergence can be described by certain pointed prehypernear spaces. To obtain a result more closer related to hypertopologies we will give the following definition.

Definition 5.4 A prehypernear space $\left(X, \mathcal{B}^{X}, N\right)$ is called surrounded, iff $N$ satisfies (sr), i.e.

(sr) $B \in \mathcal{B}^{X} \backslash\{\varnothing\}$ and $\rho \in N(B)$ imply there exists $x \in B \quad x \in \bigcap\left\{c l_{N}(F): F \in \rho\right\}$.

Remark 5.5 Here we claim that each pointed prehypernear space is surrounded, hence sected, too. (See also Definition 2.8).

Lemma 5.6 For a hypernear space $\left(X, \mathcal{B}^{X}, N\right)$ the following statements are equivalent:

(i) $\left(X, \mathcal{B}^{X}, N\right)$ is pointed;

(ii) $\left(X, \mathcal{B}^{X}, N\right)$ is surrounded.

Proof. The only remaining implication "(ii) $\Rightarrow$ (i)" will be shown now: $B \in \mathcal{B}^{X} \backslash\{\varnothing\}$ and $\rho \in N(B)$ imply the existence of $x \in B$ with $x \in \bigcap\left\{c l_{N}(F): F \in \rho\right\}$. Consequently,

$$
\left\{c l_{N}(F): F \in \rho\right\} \ll\{\{x\}\} \in N(\{x\}),
$$

hence $\left\{c l_{N}(F): F \in \rho\right\} \in N(\{x\})$ follows, and $\rho \in N(\{x\})$ results according to (hn).

Remark 5.7 Now, if we consider a bounded hypertopology, this is a psb-hull operator $h$ on a B-set $\mathcal{B}^{X}$, which additionally satisfies $\left(\mathrm{bh}_{4}\right)$, i.e.

$\left(\mathrm{bh}_{4}\right) \quad B \subset X$ and $h(B) \in \mathcal{B}^{X}$ imply $h(h(B))=h(B)$, then the corresponding category is isomorphic to the full subcategory $\boldsymbol{S R}-\boldsymbol{H N}$ of $\boldsymbol{H N}$, whose objects are the surrounded hypernear spaces. In this connexion we consider the restriction of $c l_{N}$ on the B-set $\mathcal{B}^{X}$. Conversely, for a bounded hypertopological space $\left(Y, \mathcal{B}^{Y}, t\right)$ we define the corresponding sourrounded hypernear space $\left(Y, \mathcal{B}^{Y}, M_{t}\right)$ by setting $M_{t}(\varnothing):=\{\varnothing\}$; and $M_{t}(B):=\{\rho \subset \underline{P} X: \exists x \in B x \in \bigcap\{t(F): F \in \rho\}\}$, otherwise. In the saturated case then we can recover all hypertopological spaces. So, in general it is now possible to study those closure operators not only on $\underline{P} X$, but also on arbitrary $\underline{B}$-sets even in the realm of the broader concept of hypernear spaces.

Remark 5.8 In this connexion another concept of closure operators seems to be of interest, and it is playing an important rule when considering classical nearness structures. In the following we will give some notes in this direction.

Definition 5.9 We call a prehypernear space $\left(X, \mathcal{B}^{X}, N\right)$ neartopological iff $N$ is satisfying (nt), i.e.

(nt) $B \in \mathcal{B}^{X} \backslash\{\varnothing\}$ and $\rho \in N(B)$ imply $\cap\left\{c_{N}(A): A \in \rho \cup\{B\}\right\} \neq \varnothing$.

Remark 5.10 We note that each surrounded prehypernear space is neartopological. On the other hand let be given a symmetric bounded hypertopological space $\left(Y, \mathcal{B}^{Y}, h\right)$, where in addition $h$ is satisfying (sym), i.e.

(sym) $x, z \in Y$ and $x \in h(\{z\})$ imply $z \in h(\{x\})$,

then we define the corresponding neartopological hypernear space $\left(Y, \mathcal{B}^{Y}, M^{h}\right)$ by setting: $M^{h}(\varnothing):=\{\varnothing\}$ 
and $M^{h}(B):=\{\rho \subset \underline{P} X: \cap\{h(A): A \in \rho \cup\{B\}\} \neq \varnothing\}$, otherwise. By definition $\left(Y, \mathcal{B}^{Y}, M^{h}\right)$ is automatically symmetric (see Definition 2.1. (iii)). At this point we mention the fact that symmetric hypernear spaces are always dense, which means $N$ is satisfying (d), i.e.

(d) $B \subset X$ and $c l_{N}(B) \in \mathcal{B}^{X}$ imply $N\left(c l_{N}(B)\right)=N(B)$.

This can be seen as follows: Without restriction let be $B \in \mathcal{B}^{X} \backslash\{\varnothing\}, \quad \rho \in N\left(c l_{N}(B)\right)$ implies

$$
\left\{c l_{N}(B)\right\} \cup \rho \in N\left(c l_{N}(B)\right)
$$

by hypothesis.

$$
\gamma:=\left\{c l_{N}(B)\right\} \cup\left\{c l_{N}(F): F \in \rho\right\} \ll\left\{c l_{N}(B)\right\} \cup \rho,
$$

hence $\gamma \in N\left(c l_{N}(B)\right)$ follows, and $\{B\} \cup \rho \in N\left(c l_{N}(B)\right)$ is valid according to (hn). But then

$$
\left\{c l_{N}(B)\right\} \cup(\{B\} \cup \rho) \in N(B)
$$

results, since $N$ is symmetric. Consequently, $\rho \in N(B)$ can be deduced according to $\left(\mathrm{hn}_{1}\right)$. Now, we point out that in some cases $\left(X, \mathcal{B}^{X}, N\right)$ is round which means $\mathcal{B}^{X}$ additionally satisfies (ron), i.e.

(ron) $B \in \mathcal{B}^{X}$ implies $c l_{N}(B) \in \mathcal{B}^{X}$. (see also Remark 3.9.(2)).

A detailed description of this fact will be given in some forthcoming papers. Then evidently saturated spaces are round. Analogously, we can consider roundbounded symmetric hypertopological spaces, i.e. spaces $\left(X, \mathcal{B}^{X}, h\right)$, where $\mathcal{B}^{X}$ is satisfying (rb), i.e.

(rd) $B \in \mathcal{B}^{X}$ implies $h(B) \in \mathcal{B}^{X}$.

Then the corresponding category is isomorphic to the full subcategory $R \boldsymbol{N T}-\boldsymbol{H} \boldsymbol{N}$ of $\boldsymbol{H N}$, whose objects are the round neartopological hypernear spaces. As above defined we only verify the following two statements:

(i) $\mathrm{Cl}_{M^{h}} \leq h$;

(ii) $M^{c l_{N}} \leq N$.

To (i): Let be $A \subset X$ and $x \in c_{M^{h}}(A)$, then $\{A\} \in M^{h}(\{x\})$ by definition. Hence there exists $y \in h(A)$ with $y \in t(\{x\})$. Since $t$ is symmetric we get $x \in t(\{y\})$, and $x \in t(A)$ results.

To (ii): Without restriction let be $B \in \mathcal{B}^{X} \backslash\{\varnothing\} . \rho \in M^{c_{N}}(B)$ implies the existence of $x \in c_{N}(B)$ such that $x \in \bigcap\left\{c l_{N}(F): F \in \rho\right\}$. Consequently, $\left\{c l_{N}(F): F \in \rho\right\} \ll\{\{x\}\} \in N(\{x\}) \subset N\left(c l_{N}(B)\right) \subset N(B)$, and $\rho \in N(B)$ results.

In the saturated case then we can recover all symmetric hypertopological spaces.

\section{Supernear and Paranear Spaces}

Now, based on former advisements we are going to consider two special classes of hypernear spaces, which are being fundamental in the theory of topological extensions.

Definition 6.1 We call a bonded hypernear space a supernear space and denote by $\boldsymbol{S N}$ the corresponding full subcategory of $\boldsymbol{H N}$.

Corollary 6.2 The category TOP of topological spaces and continuous maps is isomorphic to a full subcategory of $\mathbf{S N}$.

Proof. According to Example 1.3. (v), Theorem 2.6, Theorem 4.4 and Definition 5.1 we only have to verify that $N^{-}$is satisfying (hn). Now, let be $B \in \mathcal{B}^{X}, \rho \subset \underline{P} X$ with $\left\{c_{N^{-}}(F): F \in \rho\right\} \in N^{-}(B)$, hence

$$
B \in \sec \left\{\overline{c l_{N^{-}}(F): F \in \rho}\right\} \text {. }
$$

For $F \in \rho$ we have $B \cap \overline{c_{N^{-}}(F)} \neq \varnothing$. But $c l_{N^{-}}(F)=\bar{F}$ is valid. Since "-” is a topological closure operator we get $\overline{c l_{N}-F}=\bar{F}$, and consequently $\rho \in N^{-}(B)$ results.

Corollary 6.3 The category STOP of supertopological spaces and continuous maps is isomorphic to a subcategory of $\mathbf{S N}$.

Proof. The reader is referred to Remark 3.7, Theorem 3.8 and Remark 4.2 respectively.

Remark 6.4 $b$-proximities (see Definition 3.10) are playing an important rule when considering topological extensions (see Remark 3.9). In this connexion we are now giving two special cases of them. First of all we call a b-proximity space $\left(X, \mathcal{B}^{X}, \delta\right)$ a preLEADER space iff $\delta$ in addition satisfies $\left(\mathrm{bp}_{5}\right)$, i.e. 
$\left(\mathrm{bp}_{5}\right) B \in \mathcal{B}^{X}$ and $B \delta A$ with $A \subset c_{\delta}(C)$ imply $B \delta C$, where $c l_{\delta}(C):=\{x \in X:\{x\} \delta C\}$.

By $\boldsymbol{p L E S P}$ we denote the corresponding full subcategory of $\boldsymbol{b}$-PROX.

In the saturated case (if $X \in \mathcal{B}^{X}$ ) LEADER proximity spaces then can be recovered as special objects.

Corollary 6.5 The category pLESP is isomorphic to a full subcategory of $\mathbf{S N}$.

Proof. According to Example 1.3. (vi), Remark 3.11 and Theorem 3.13 respectively it remains to verify that $N_{\delta}$ satisfies (hn) and $p_{M}\left(\mathrm{bp}_{5}\right)$ respectively.

To (hn): $B \in \mathcal{B}^{X}, \rho \subset \underline{P} X$ and $\gamma:=\left\{c l_{N_{\delta}}(F): F \in \rho\right\} \in N_{\delta}(B)$ imply $\gamma \subset \delta(B)$. We have to verify that $\rho \subset \delta(B)$. So let be $F \in \rho$, hence $c l_{N_{\delta}}(F) \in \delta(B)$, and consequently $B \delta c l_{N_{\delta}}(F)$ is valid. The inclusion $c l_{N_{\delta}}(F) \subset c l_{\delta}(F)$ holds, because $x \in c l_{N_{\delta}}(F)$ implies $\{F\} \in N_{\delta}(\{x\})$, hence $\{F\} \subset \delta(\{x\})$, and $\{x\} \delta F$ results, showing that $x \in c_{\delta}(F)$ is valid. According to $\left(\mathrm{bp}_{5}\right)$ we get $B \delta F$, and the proposed inclusion holds.

To (bps): Conversely, let be $B \in \mathcal{B}^{X}$ and $B p_{M} A$ with $A \subset c l_{p_{M}}(C)$, we have to verify $B p_{M} C$.

By hypothesis $\{A\} \in M(B)$ is valid. $\left\{c l_{M}(C)\right\} \ll\{A\}$, since $c l_{p_{M}}(C) \subset c l_{M}(C)$. Because $x \in c l_{p_{M}}(C)$ implies $\{x\} p_{M} C,\{C\} \in M(\{x\})$ leads us to the statement $x \in c l_{M}(C)$. According to $\left(\mathrm{hn}_{1}\right)$ we obtain $\left\{c l_{M}(C)\right\} \in M(B)$, and $\{C\} \in M(B)$ results by axiom (hn). Consequently $B p_{M} C$ is valid.

Remark 6.6 At this point we note that certain supernear spaces are in one-to-one correspondence to strict topological extensions which we study in a forthcoming paper. Here, we will examine the case if a symmetric topological extension is presumed (see Example 1.7. (ii)). In this connexion bunch-determined nearness and certain preLODATO spaces are playing an important role. Now, we will give the definition of a preLODATO space:

Definition 6.7 A preLEADER space $\left(X, \mathcal{B}^{X}, \delta\right)$ is called a preLODATO space iff $\delta$ in addition satisfies the following axioms, i.e.

$\left(\mathrm{bp}_{6}\right) \quad B_{1} \cup B_{2} \in \mathcal{B}^{X}$ and $\left(B_{1} \cup B_{2}\right) \delta A$ imply $B_{1} \delta A$ or $B_{2} \delta A$;

$\left(\mathrm{bp}_{6}\right) \quad B, A \subset X, c l_{\delta}(B) \in \mathcal{B}^{X}$ and $c l_{\delta}(B) \delta A$ imply $B \delta A$;

(bp $)_{6} B_{1}, B_{2} \in \mathcal{B}^{X}$ and $B_{1} \delta B_{2}$ imply $B_{2} \delta B_{1}$.

By $\boldsymbol{p L O S P}$ we denote the corresponding full subcategory of $\boldsymbol{p L E S P}$.

Remark 6.8 In the saturated case LODATO proximity spaces then can be recovered as special objects. Moreover, we note that each b-supertopological space then can be regarded as special preLODATO space. A slight specialization lead us to the so-called LODATO space by adding the axiom $\left(\mathrm{bp}_{9}\right)$, i.e.

(bpg) $B \in \mathcal{B}^{X}$ implies $c l_{\delta}(B) \in \mathcal{B}^{X}$.

Once again, in the saturated case the two definitions coincide, and LODATO proximity spaces then can be recovered as special objects.

But in general the two definitions differ, and the reader is referred to Remark 3.9 in connexion with Remark 5.10. In a forthcoming paper we will show that the corresponding category $\mathbf{L O S P}$ of LODATO spaces can be regarded as a full subcategory of $\boldsymbol{S N}$, whose objects are symmetric. On the other hand nearness also leads us to a certain symmetric supernear space, hence we give the following definition.

Definition 6.9. A symmetric supernear space is called a paranear space and we denote by $\boldsymbol{P N}$ the corresponding full subcategory of $S N$.

Theorem 6.10. The category NEAR of nearness spaces and related maps is isomorphic to a full subcategory of $\mathbf{P N}$.

Proof. According to Example 1.3. (ii) and Theorem 4.5 respectively it remains to verify that $N_{\xi}$ satisfies (hn) and $\eta_{M}$ the nearness axiom.

To (hn): Without restriction let be $B \in \mathcal{B}^{X} \backslash\{\varnothing\}, \rho \in \underline{P} X$ and $\left\{c l_{N_{\xi}}(F): F \in \rho\right\} \in N_{\xi}(B)$, hence

$$
\gamma:=\{B\} \cup\left\{c l_{N_{\xi}}(F): F \in \rho\right\} \in \xi .
$$

But $\left\{c l_{\xi}(B)\right\} \cup\left\{c l_{\xi}(F): F \in \rho\right\} \ll \gamma$, because for $F \in \rho$ and $x \in c l_{N_{\xi}}(F)$ we have $\{F\} \in N_{\xi}(\{x\})$, hence $\{\{x\} \cup\{F\}\} \in \xi$ is valid. Consequently $\{\{x\}, F\} \in \xi$ results which shows $x \in c_{\xi}(F)$. Since $\xi$ satisfies the nearness axiom: we get $\{B\} \cup \rho \in \xi$, and $\rho \in N_{\xi}(B)$ results. Conversely, let be $\left\{c l_{\eta_{M}}(F): F \in \rho\right\} \in \eta_{M}$ for $\rho \subset \underline{P} X$. We have to verify $\rho \in \eta_{M}$. So let be $A \in \rho$, our goal is to show $\rho \in M(A)$. By hypothesis we get $\left\{c l_{\eta_{M}}(F): F \in \rho\right\} \in M\left(c l_{\eta_{M}}(A)\right)$. But $c l_{\eta_{M}}(A) \subset c l_{M}(A)$, since $x \in c l_{\eta_{M}}(A)$ implies $\{\{x\}, A\} \in \eta_{M}$, hence 
$\{\{x\}, A\} \in M(\{x\})$ follows, and $\{A\} \in M(\{x\})$ is valid, which shows $x \in c_{M}(A)$. Consequently, $\gamma:=\left\{c l_{\eta_{M}}(F): F \in \rho\right\} \in M\left(c l_{M}(A)\right)$ with $\left\{c l_{M}(F): F \in \rho\right\} \ll \gamma$ is valid according to (is) of Definition 2.4. Since $M$ is dense (see Remark 5.10) we get $\left\{c l_{M}(F): F \in \rho\right\} \in M(A)$ according to $\left(\mathrm{hn}_{1}\right)$. But then $\rho \in M(A)$ follows by (hn), which concludes the proof.

Corollary 6.11. For a saturated paranear space $\left(X, \mathcal{B}^{X}, N\right)$ the following statements are equivalent:

(i) $\eta_{M}$ is topological nearness;

(ii) $\left(X, \mathcal{B}^{X}, N\right)$ is neartopological.

Proof. evident according to Remark 5.10.

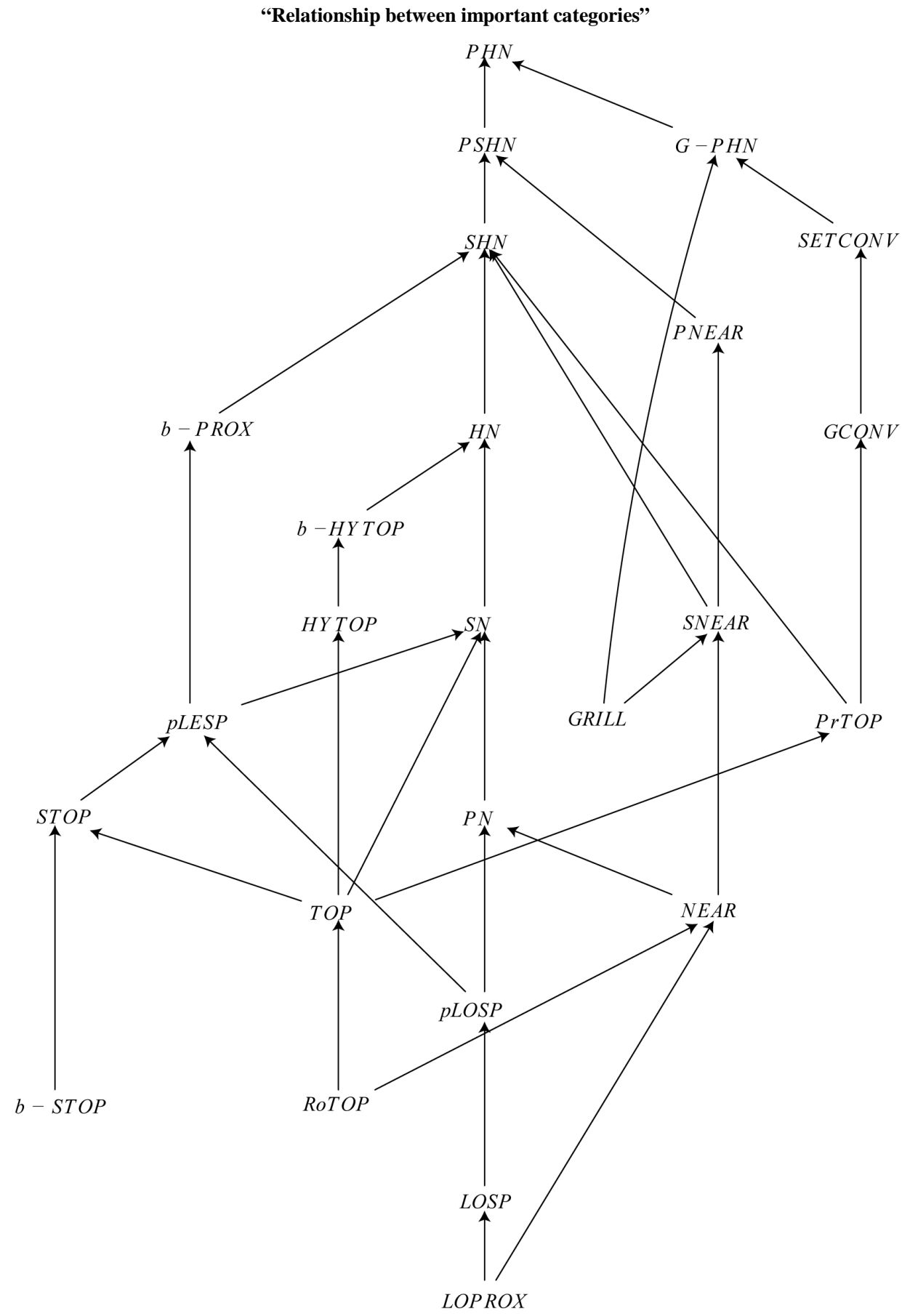




\section{Topological Extensions and Their Corresponding Paranear Spaces}

Taking into account Example 1.7.(ii), Remark 3.9, 6.6 and 6.8 respectively we will now consider the problem for finding a one-to-one correspondence between certain topological extensions and their related paranear spaces. In this connexion we point out that certain grill-spaces come into play.

Definition 7.1 Let be given a supernear space $\left(X, \mathcal{B}^{X}, N\right)$. For $B \in \mathcal{B}^{X} \backslash\{\varnothing\}, \mathcal{C} \in \operatorname{GRL}(X)$ is called a $B$-clan in $N$ iff it satisfies

$\left(\mathrm{cla}_{1}\right) \quad B \in \mathcal{C} \in N(B)$;

$\left(\mathrm{cla}_{2}\right) \quad A \in \mathcal{C}$ and $A \subset C l_{N}(F)$ imply $F \in \mathcal{C}$.

Remark 7.2 For a supernear space $\left(X, \mathcal{B}^{X}, N\right)$ and each $B \in \mathcal{B}^{X}$ with $x \in B x_{N}:=\left\{A \subset X: x \in c l_{N}(A)\right\}$ is a B-clan in $N$.

Definitions 7.3 A supernear space respectively paranear space $\left(X, \mathcal{B}^{X}, N\right)$ is called superclan space respectively paraclan space iff $N$ satisfies (cla), i.e.

(cla) $B \in \mathcal{B}^{X} \backslash\{\varnothing\}$ and $\rho \in N(B)$ imply the existence of a B-clan $\mathcal{C}$ in $N$ with $\rho \subset \mathcal{C}$.

Remark 7.4 In giving some examples we note that each surrounded supernear space is a superclan space, and each neartopological paranear space is a paraclan space. This is analogical valid for the spaces considered in 1.7.

Proof of Example 1.7. (ii)

First, we prove the equality of the corresponding closure operators. So, let be $A \in \underline{P} X$ and $x \in c l_{X}(A)$, then by $\left(\operatorname{tx}_{1}\right) e(x) \in c l_{Y}(e[A])$ with $e(x) \in C l_{Y}(\{e(x)\})$, hence $\{A\} \in N^{e}(\{x\})$ follows, which shows $x \in C l_{N^{e}}(A)$. Conversely, let $x \in C l_{N^{e}}(A)$, hence $\{A\} \in N^{e}(\{x\})$ follows, which implies the existence of $y \in c l_{Y}(\{e(x)\})$ with $y \in c l_{Y}(e[A])$. But now, $e(x) \in c l_{Y}(\{y\})$ holds, because the presumed extension is symmetric. Consequently, $e(x) \in c l_{Y}(e[A])$ follows, which shows that $x \in c l_{X}(A)$ according to ( $\left.\operatorname{tx}_{1}\right)$. Alltogether, the equality now results. Secondly, it is easy to verify that $\left(X, \mathcal{B}^{X}, N^{e}\right)$ fulfills the axioms for being a semihypernear space. $N^{\mathrm{e}}$ is symmetric, since $\rho \in N^{e}(B)$ for $B \in \mathcal{B}^{X} \backslash\{\varnothing\}$ implies the existence of $y \in c l_{Y}(e[B])$ with $y \in \bigcap\left\{c l_{Y}(e[A]): A \in \rho\right\}$, hence $\{B\} \cup \rho \in N^{e}(B)$ follows. Now, $F \in \rho \cap \mathcal{B}^{X}$ implies $\{B\} \cup \rho \in N^{e}(F)$, since $y \in c l_{Y}(e[B]) \cap c l_{Y}(e[F])$ by supposition, hence $N^{e}$ symmetric. $\left(X, \mathcal{B}^{X}, N^{e}\right)$ is a supernear space, because $\rho \subset \underline{P} X$ and $\left\{c l_{N^{e}}(F): F \in \rho\right\}=: \mathcal{M} \in N^{e}(B)$ imply the existence of $y \in c l_{Y}(e[B])$ with $y \in \bigcap\left\{c l_{Y}(e[A]): A \in \mathcal{M}\right\} . F \in \rho$ implies $c l_{N^{e}}(F) \in \mathcal{M}$, hence

$$
y \in c l_{Y}\left(e\left[c l_{N^{e}}(F)\right]\right)=c l_{Y}\left(e\left[c l_{X}(F)\right]\right) \subset c l_{Y}\left(c l_{Y}(e[F])\right) \subset c l_{Y}(e[F]),
$$

consequently $\rho \in N^{e}(B)$ follows, which shows that $\left(X, \mathcal{B}^{X}, N^{e}\right)$ is a paranearness space. It remains to prove $N^{\mathrm{e}}$ satisfies the axiom (cla). For $B \in \mathcal{B}^{X} \backslash\{\varnothing\}$ let be $\rho \in N^{e}(B)$, hence $y \in \bigcap\left\{c l_{Y}(e[A]): A \in \rho\right\}$ for some $y \in \operatorname{cl}_{Y}(e[B])$. We set $\mathcal{C}:=\left\{T \subset X: y \in \operatorname{cl}_{Y}(e[T])\right\}$, consequently $\mathcal{C}$ is the desired $B$-clan in $N^{e}$ proving that $\left(X, \mathcal{B}^{X}, N^{e}\right)$ is a paraclan space.

Convention 7.5 We denote by SY-TEXT the full subcategory of $\boldsymbol{T E X T}$, whose objects are the symmetric topological extensions and by $\boldsymbol{C L A - P N}$ the full subcategory of $\boldsymbol{P N}$, whose objects are the paraclan spaces.

Theorem 7.6 Let $F:$ SY-TEXT $\rightarrow$ CLA-PN be defined by:

(a) For a SY-TEXT-object $\left(e, \mathcal{B}^{X}, Y\right)$ we put $F\left(e, \mathcal{B}^{X}, Y\right):=\left(X, \mathcal{B}^{X}, N^{e}\right)$;

(b) for a TEXT-morphism $(f, g):\left(e, \mathcal{B}^{X}, Y\right) \rightarrow\left(e^{\prime}, \mathcal{B}^{X^{\prime}}, Y^{\prime}\right)$ we put $F(f, g):=f$.

Then $F: S Y-T E X T \rightarrow C L A-P N$ is a functor.

Proof. We already know that the image of $F$ lies in $\boldsymbol{C L A}-\boldsymbol{P N}$. Now, let $(f, g):\left(e, \mathcal{B}^{X}, Y\right) \rightarrow\left(e^{\prime}, \mathcal{B}^{X^{\prime}}, Y^{\prime}\right)$ be a TEXT-morphism; it has to be shown that $f$ preserves B-near collections for each $B \in \mathcal{B}^{X}$. Without restriction let $B \in \mathcal{B}^{X} \backslash\{\varnothing\}$ and $\rho \in N^{e}(B)$, hence we can choose $y \in c l_{Y}(e[B])$ with $y \in \bigcap\left\{c l_{Y}(e[A]): A \in \rho\right\}$. Our goal is to verify the existence of $z \in c l_{Y^{\prime}}\left(e^{\prime}[f(B)]\right)$ such that $z \in \bigcap\left\{c l_{Y^{\prime}}\left(e^{\prime} f[A]\right): A \in \rho\right\}$. By hypothesis we have $g(y) \in g\left(c l_{Y}(e[B])\right)$, consequently $g(y) \in c l_{Y^{\prime}}\left(e^{\prime}[f[B]]\right)$ results, since $(f, g)$ is a TEXT-morphism by assumption. Now, consider some $A \in \rho$, because $y \in c l_{Y}(e[A])$, we have $g(y) \in c l_{Y^{\prime}}\left(e^{\prime}[f[A]]\right)$, which results in $f \rho \in N^{e^{\prime}}(f[B])$. 


\section{Strict Topological Extensions}

Remark 8.1 In the previous section we have found a functor from SY-TEXT to CLA-PN. Now, we are going to introduce a related one in the opposite direction.

Lemma 8.2 Let $\left(X, \mathcal{B}^{X}, N\right)$ be a paranear space. We set $X^{C}:=\{\mathcal{C} \subset \underline{P} X: \mathcal{C}\}$ is a B-clan in $N$ for some $B \in \mathcal{B}^{X} \backslash\{\varnothing\}$, and for each $A^{C} \subset X^{C}$ we put:

$$
c l_{X^{C}}\left(A^{C}\right):=\left\{\mathcal{C} \in X^{C}: \Delta A^{C} \subset \mathcal{C}\right\}
$$

where $\Delta A^{C}:=\left\{F \subset X: \forall \mathcal{C} \in A^{C} F \in \mathcal{C}\right\}$. (By convention $\Delta A^{C}=\underline{P} X$ if $A^{C}=\varnothing$ ). Then $c l_{X^{C}}: \underline{P} X^{C} \rightarrow \underline{P} X^{C}$ is a topological closure operator.

Proof. We first note that $c_{X^{C}}(\varnothing)=\varnothing$, since $\varnothing \notin \mathcal{C}$ for each $\mathcal{C} \in X^{C}$. Let $A^{C}$ be a subset of $X^{C}$ and consider $\mathcal{C} \in A^{C}$. Then $F \in \Delta A^{C}$ implies $F \in \mathcal{C}$, hence $A^{C} \subset c l_{X^{C}}\left(A^{C}\right)$. Now, let be $A_{1}^{C} \subset A_{2}^{C} \subset X^{C}$. Then, $\Delta A_{2}^{C} \subset \Delta A_{1}^{C}$, which implies $c l_{X^{C}}\left(A_{1}^{C}\right) \subset c l_{X^{C}}\left(A_{2}^{C}\right)$. For arbitrary subsets $A_{1}^{C}, A_{2}^{C} \subset X^{C}$ we consider an element $\mathcal{C} \in X^{C}$ such that $\mathcal{C} \notin c l_{X^{C}}\left(A_{1}^{C}\right) \cup c l_{X^{C}}\left(A_{2}^{C}\right)$. Then we have $\Delta A_{1}^{C} \not \subset \mathcal{C}$ and $\Delta A_{2}^{C} \not \subset \mathcal{C}$. Choose $F_{1} \in \Delta A_{1}^{C}$ with $F \notin \mathcal{C}$ and $F_{2} \in \Delta A_{2}^{C}$ with $F_{2} \notin \mathcal{C}$. Because $\mathcal{C} \in \operatorname{GRL}(X)$ we get $F_{1} \cup F_{2} \notin \mathcal{C}$. On the other hand, $F_{1} \cup F_{2} \in \Delta A_{1}^{C} \cup \Delta A_{2}^{C} \subset \Delta\left(A_{1}^{C} \cup A_{2}^{C}\right)$ implies $\mathcal{C} \notin C l_{X^{C}}\left(A_{1}^{C} \cup A_{2}^{C}\right)$. At last, let $C$ be an element of $\mathrm{cl}_{X^{C}}\left(c l_{X^{C}}\left(A^{C}\right)\right)$ and suppose $\mathcal{C} \notin C l_{X^{C}}\left(A^{C}\right)$. Choose $F \in \Delta A^{C}$ with $F \notin \mathcal{C}$. By assumption we have $\Delta c l_{X^{C}}\left(A^{C}\right) \subset \mathcal{C}$, hence $F \notin \Delta c l_{X^{C}}\left(A^{C}\right)$. Consequently there exists $\mathcal{D} \in c l_{X^{C}}\left(A^{C}\right)$ with $F \notin \mathcal{D}$ But this implies $\Delta A^{C} \subset \mathcal{D}$, and $F \in \mathcal{D}$ results, which leads us to a contradiction.

Theorem 8.3 For paranear spaces $\left(X, \mathcal{B}^{X}, N\right),\left(Y, \mathcal{B}^{Y}, M\right)$ let $f: X \rightarrow Y$ be a hn-map. Define a function $f^{C}: X^{C} \rightarrow Y^{C}$ by setting for each $\mathcal{C} \in X^{C}:$

$$
f^{C}(\mathcal{C}):=\left\{D \subset Y: f^{-1}\left[C l_{M}(D)\right] \in \mathcal{C}\right\}
$$

Then the following statements are valid:

(1) $f^{C}$ is a continuous map from $\left(X^{C}, c l_{X^{C}}\right)$ to $\left(Y^{C}, c l_{Y^{C}}\right)$;

(2) The composites $f^{C} \circ e_{X}$ and $e_{Y} \circ f$ coincide, where $e_{X}: X \rightarrow X^{C}$ denotes the function which assigns the $\{x\}$-clan $x_{N}$ to each $x \in X$.

Proof. First, let $\mathcal{C}$ be a $B$-clan in $N$. We will show that $f^{C}(\mathcal{C})$ is a $f[B]$-clan in $M$. It is easy to verify that $f^{C}(\mathcal{C}) \in \operatorname{GRL}(Y)$, which satisfies (cla ${ }_{2}$ ) in Definition 7.1. In order to establish (cla $)_{1}$ ) we observe that $B \in \mathcal{C} \in N(B)$ by hypothesis. We will now verify that

$$
\left\{c l_{M}(D): D \in f^{C}(\mathcal{C})\right\} \ll f \mathcal{C}
$$

(Note, that $f$ is a hn-map by assumption.) For any $D \in f^{C}(\mathcal{C})$ we have $f^{-1}\left[c l_{M}(D)\right] \in \mathcal{C}$, hence

$$
c l_{M}(D) \supset f\left[f^{-1}\left[C l_{M}(D)\right]\right] \in f^{C} \mathcal{C} .
$$

Since $B \in \mathcal{C}$ and $B \subset f^{-1}[f[B]] \subset f^{-1}\left[C l_{M}(f[B])\right]$ we get $f[B] \in f^{C}(\mathcal{C})$, and all together we conclude that $f^{C}(\mathcal{C})$ defines a $f[B]$-clan in $M$. Consequently, $f^{C}(\mathcal{C}) \in Y^{C}$ results.

To (1): Let $A^{C} \subset X^{C}, \mathcal{C} \in c_{X^{C}}\left(A^{C}\right)$ and suppose $f^{C}(\mathcal{C}) \notin c l_{Y^{C}}\left(f^{C}\left[A^{C}\right]\right)$. Then $\Delta f^{C}\left[A^{C}\right] \not \subset f^{C}(\mathcal{C})$, hence $D \notin f^{C}(\mathcal{C})$ for some $D \in \Delta f^{C}\left[A^{C}\right]$, which means $f^{-1}\left[c l_{M}(D)\right] \notin \mathcal{C}$. Since $\Delta A^{C} \subset \mathcal{C}$, we get $f^{-1}\left[c_{M}(D)\right] \notin \mathcal{D}$ for some $\mathcal{D} \in A^{C}$. Consequently $D \notin f^{C}(\mathcal{D})$ results, which leads us to a contradiction, because $D \in \Delta f^{C}\left[A^{C}\right]$ is valid.

To (2): Let $x$ be an element of $X$. We will prove the validity of $f^{C}\left(e_{X}(x)\right)=e_{Y}(f(x))$. To this end, let $D \in e_{Y}(f(x))$. Then, $f(x) \in c l_{M}(D)$, hence $x \in f^{-1}\left[c l_{M}(D)\right]$, and consequently $f^{-1}\left[c l_{M}(D)\right] \in x_{N}=e_{X}(x)$. Thus $D \in f^{C}\left(e_{X}(x)\right)$, proving the inclusion $e_{Y}(f(x)) \subset f^{C}\left(e_{X}(x)\right)$. Since $e_{Y}(f(x))$ is maximal with respect to $(M(\{f(x)\} \backslash\{\varnothing\}, \subset))$ and moreover $\left\{c l_{M}(D): D \in f^{C}\left(e_{X}(x)\right)\right\} \ll f e_{X}(x)$, since by hypothesis $f$ is a hn-map, we obtain the desired equality. 
Theorem 8.4 We obtain a functor G: CLA-PN to SY-TEXT by setting:

(a) $G\left(X, \mathcal{B}^{X}, N\right):=\left(e_{X}, \mathcal{B}^{X}, X^{C}\right)$ for any paraclan space $\left(X, \mathcal{B}^{X}, N\right)$ with $X:=\left(X, c_{N}\right)$ and $X^{C}:=\left(X^{C}, c l_{X^{C}}\right)$;

(b) $G(f):=\left(f, f^{C}\right)$ for any hn-map $f:\left(X, \mathcal{B}^{X}, N\right) \rightarrow\left(Y, \mathcal{B}^{Y}, M\right)$.

Proof. With respect to Corollary 6.2 it is straight forward to verify that $c l_{N}$ is a topological closure operator on $X$. We also have the topological closure operator $c l_{X^{C}}$ on $X^{C}$. Therefore we obtain topological spaces with $\underline{B}$-set $\mathcal{B}^{X}$, and $e_{X}: X \rightarrow X^{C}$ is a continuous map, which can be seen as follows: Let $\mathcal{C} \in e_{X}\left[c_{N}(A)\right]$ for $A \subset X$, we have to verify that $\Delta e_{X}[A] \subset \mathcal{C} . F \in \Delta e_{X}[A]$ implies $A \subset c l_{N}(F)$ and $\mathcal{C}=e_{X}(x)$ for some $x \in C l_{N}(A)$ by supposition. Consequently, $x \in C l_{N}(F)$ follows which shows $F \in X_{N}=e_{X}(x)=\mathcal{C}$. To establish $\left(\mathrm{tx}_{1}\right)$, let $A$ be a subset of $X$ and suppose $x \in c l_{N}(A)$. Then we get $\Delta e_{X}[A] \subset e_{X}(x)$, hence $e_{X}(x) \subset c l_{X^{C}}\left(e_{X}[A]\right)$ which means that $x \in e_{X}^{-1}\left[c l_{X^{C}}\left(e_{X}[A]\right)\right]$. Conversely, let $x$ be an element of $e_{X}^{-1}\left[c l_{X^{C}}\left(e_{X}[A]\right)\right]$. Then by definition we have $e_{X}(x) \in c l_{X^{C}}\left(e_{X}[A]\right)$, and consequently $\Delta e_{X}[A] \subset e_{X}(x)$. This implies $A \in e_{X}(x)$, which means $x \in c_{N}(A)$. To establish $\left(\mathrm{tx}_{2}\right)$, let be $\mathcal{C} \in X^{C}$ and suppose $\mathcal{C} \notin C l_{X^{C}}\left(e_{X}[X]\right)$. By definition we get $\Delta e_{X}[X] \not \subset \mathcal{C}$, so that there exists a set $F \in \Delta e_{X}[X]$ with $F \notin \mathcal{C}$. But $X \subset c_{N}(F)$ follows. Since $B \in \mathcal{C}$ for some $B \in \mathcal{B}^{X} \backslash\{\varnothing\}$, we get $c l_{N}(F) \in \mathcal{C}$, hence $F \in \mathcal{C}$, because $\mathcal{C}$ satisfies $\left(\mathrm{cla}_{2}\right)$. But this is a contradiction, and thus $\mathcal{C} \in c_{X^{C}}\left(e_{X}[X]\right)$ is valid. In showing $\left(e_{X}, \mathcal{B}^{X}, X^{C}\right)$ is symmetric let $x$ be an element of $X$ such that $\mathcal{C} \in c_{X^{C}}\left(\left\{e_{x}(x)\right\}\right)$. We have to prove $e_{X}(x) \in c l_{X^{C}}(\{\mathcal{C}\})$. By hypothesis we have $x_{N}=e_{X}(x) \subset \mathcal{C}$ and moreover $B \in \mathcal{C} \in N(B)$ for some $B \in \mathcal{B}^{X} \backslash\{\varnothing\}$. Since $\{x\} \in \mathcal{C}$ and $N$ is symmetric we get $\{B\} \cup \mathcal{C} \in N(\{x\})$ with $\mathcal{C} \ll\{B\} \cup \mathcal{C}$, hence $\mathcal{C} \in N(\{x\})$ follows according to (hn ${ }_{1}$ ). But $x_{N}$ is maximal with respect to $(N(\{x\}) \backslash\{\varnothing\}, \subset)$, which means that $\mathcal{C}$ coincides with $x_{N}=e_{X}(x)$. By hypothesis $f:\left(X, \mathcal{B}^{X}, N\right) \rightarrow\left(Y \mathcal{B}^{Y}, M\right)$ is a hn-map, so that $f$ is continuous and bounded with respect to the given $\underline{B}$-sets and corresponding closure operators. It remains to prove that the following diagram commutes:

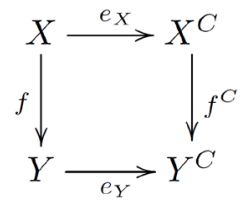

To this end let $x$ be an element of $X$. We must show $\left(f^{c} \circ e_{X}\right)(x)=\left(e_{Y} \circ f\right)(x)$.

“ $\subset$ ” $D \in\left(f^{c} \circ e_{X}\right)(x)$ implies $D \in f^{C}\left(x_{N}\right)$, which means $f^{-1}\left[c l_{M}(D)\right] \in x_{N}$, hence

$$
x \in c l_{N}\left(f^{-1}\left[c l_{M}(D)\right]\right)
$$

Since $f$ is continuous we have $f(x) \in c l_{M}\left(f\left[f^{-1}\left[c l_{M}(D)\right]\right]\right)$ and $D \in f(x)_{M}=e_{Y}(f(x))$ follows.

“ $\supset$ ” $D \in e_{Y}(f(x))$ implies $f(x) \in c l_{M}(D)$, hence $x \in f^{-1}\left[c l_{M}(D)\right]$ follows and consequently

$x \in c l_{N}\left(f^{-1}\left[c l_{M}(D)\right]\right)$. Thus, $f^{-1}\left[c l_{M}(D)\right] \in e_{X}(x)$, which means $D \in f^{C}\left(e_{X}(x)\right)$.

Finally, this establishes that the composition of hn-maps is preserved by $G$. At last we will show that the image of $G$ also is contained in STR-TEXT, whose objects are the strict topological extensions. Consider $\mathcal{C} \in X^{C}$ and let $A^{C}$ be closed in $X^{C}$ with $\mathcal{C} \notin A^{C}$. Then $\mathcal{C} \notin C l l_{X^{C}}\left(A^{C}\right)$, hence $\Delta A^{C} \not \mathcal{C}$. We can find some $F \in \Delta A^{C}$ such that $F \notin \mathcal{C}$. Now, for each $\mathcal{D} \in A^{C}$ we have $\stackrel{X^{C}}{F} \in \mathcal{D}$, which implies $\Delta e_{X}[F] \subset \mathcal{D}$, and therefore we conclude $\mathcal{D} \in c_{X^{C}}\left(e_{X}[F]\right)$. On the other hand since $F \notin \mathcal{C}$, we have $\Delta e_{X}[F] \not \subset \mathcal{C}$, hence $\mathcal{C} \notin c l_{X^{C}}\left(e_{X}[F]\right)$, which put an end of this.

Theorem 8.5 Let F:SY-TEXT $\rightarrow$ CLA-PN and G:CLA-PN $\rightarrow$ SY-TEXT be the above defined functors. For each object $\left(X, \mathcal{B}^{X}, N\right)$ of $\mathbf{C L A - P N}$ let $t_{\left(\mathcal{B}^{X}, N\right)}$ denote the identity map

$$
i d_{X}: F\left(G\left(X, \mathcal{B}^{X}, N\right)\right) \rightarrow\left(X, \mathcal{B}^{X}, N\right)
$$

Then $t: F \circ G \rightarrow \underline{1}_{C L A-P N}$ is natural equivalence from $F \circ G$ to the identity functor $\underline{1}_{C L A-P N}$, i.e. 


$$
i d_{X}: F\left(G\left(X, \mathcal{B}^{X}, N\right)\right) \rightarrow\left(X, \mathcal{B}^{X}, N\right)
$$

is a hn-map in both directions for each object $\left(X, \mathcal{B}^{X}, N\right)$, and the following diagram commutes for each hn-map $f:\left(X, \mathcal{B}^{X}, N\right) \rightarrow\left(Y, \mathcal{B}^{Y}, M\right)$ :

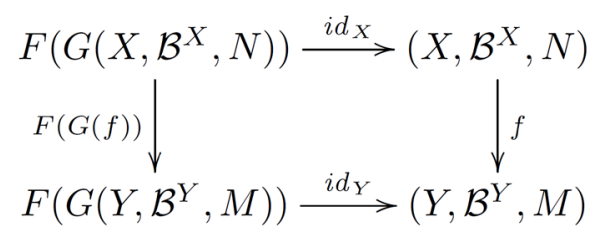

Proof. The commutativity of the diagram is obvious, because of $F(G(f))=f$. It remains to prove that

$$
i d_{X}: F\left(G\left(X, \mathcal{B}^{X}, N\right)\right) \rightarrow\left(X, \mathcal{B}^{X}, N\right)
$$

is a hn-map in both directions. To fix the notation let $N_{1}$ be such that

$$
F\left(G\left(X, \mathcal{B}^{X}, N\right)\right)=F\left(e_{X}, \mathcal{B}^{X}, X^{C}\right)=\left(X, \mathcal{B}^{X}, N_{1}\right) .
$$

It suffices to show that for each $B \in \mathcal{B}^{X} \backslash\{\varnothing\}$ we have $N_{1}(B) \subset N(B) \subset N_{1}(B)$. To this end assume $\rho \in N_{1}(B)$, then there exists $\mathcal{C} \in c_{X^{C}}\left(e_{X}[B]\right)$ such that $\mathcal{C} \in \bigcap\left\{c l_{X^{C}}\left(e_{X}[A]\right): A \in \rho\right\}$, hence $\Delta e_{X}[B] \subset \mathcal{C}$. We get $B \in \mathcal{C}$ and $\mathcal{C} \in N\left(B_{1}\right)$ for some $B \in \mathcal{B}^{X} \backslash\{\varnothing\}$. Since $N$ is symmetric, we obtain $\left\{B_{1}\right\} \cup \mathcal{C} \in N(B)$ with $\mathcal{C} \ll\left\{B_{1}\right\} \cup \mathcal{C}$, hence $\mathcal{C} \in N(B)$. But $A \in \rho$ implies $\mathcal{C} \in c_{X^{C}}\left(e_{X}[A]\right)$, hence $\Delta e_{X}[A] \subset \mathcal{C}$ with $A \in \Delta e_{X}[A]$. Now $A \in \mathcal{C}$ results, which shows $\rho \in N(B)$. Conversely, let $\rho \in N(B)$. Since $\left(X, \mathcal{B}^{X}, N\right)$ is a paraclan space we can choose a $B$-clan in $N$ such that $\rho \subset \mathcal{C}$. In order to show $\rho \in N_{1}(B)$ we need to verify

(1) $\mathcal{C} \in C l_{X^{C}}\left(e_{X}[B]\right)$;

(2) $A \in \rho$ implies $\mathcal{C} \in c_{X^{C}}\left(e_{X}[A]\right)$.

To (1): By definition of $C^{X} l_{X}^{C}$ it suffices to establish $\Delta e_{X}[B] \subset \mathcal{C}$. So let $D$ be an element of $\Delta e_{X}[B]$, hence $B \subset c l_{N}(D)$ follows which implies $c l_{N}(D) \in \mathcal{C}$. But $\mathcal{C}$ is $B$-clan in $N$, consequently we get $D \in \mathcal{C}$.

To (2): Let $A$ be an element of $\rho$ and $D$ be an element of $\Delta e_{X}[A]$, hence $A \subset c l_{N}(D)$. Since $A \in \mathcal{C}$ by hypothesis, we get $c l_{N}(D) \in \mathcal{C}$ and analogously as above we infer $D \in C$, which concludes the proof.

Remark 8.6 Making the theorem more transparent we claim that a paranear space is a paraclan space if it can be embedded in a topological space $Y$ such that the $B$-near collections are characterized by the fact that the closures of its members meet in $Y$. Therefore this theorem generalize in one direction the Bentleycharacterization of bunch-determined nearness spaces, in another the description of Doitchinov's $b$-supertopologies by compactly determined topological extensions and moreover the analogous existing correspondence respected to LODATO spaces involving the famous theorem of LODATO.

Corollary 8.7 If $\left(X, \mathcal{B}^{X}, N\right)$ is separated that means $N$ satisfies (sep), i.e.

(sep) $x, z \in X$ and $\{\{Z\}\} \in N(\{x\})$ imply $x=z$, then $e_{X}: X \rightarrow X^{C}$ is injective. Conversely, for a $T_{1}$ extension $\left(e, \mathcal{B}^{X}, Y\right)$, where $e$ is a topological embedding and $\left(Y=Y, c l_{Y}\right)$ a $T_{1}$-space, then $\left(X, \mathcal{B}^{X}, N^{e}\right)$ is separated.

\section{References}

[1] Herrlich, H. (1974) A Concept of Nearness. General Topology and Its Applications, 5, 191-212. http://dx.doi.org/10.1016/0016-660X(74)90021-X

[2] Leseberg, D. (2008) Comprehensive Convergence. IJPAM, 43, 371-392.

[3] Wyler, O. (1988) Convergence of Flters and Ultraflters to Subsets. Lecture Notes in Computer Science, 393, 340-350.

[4] Preuss, G. (2002) Foundations of Topology. An Approach to Convenient Topology. Kluwer, Dordrecht.

[5] Čech, E. (1966) Topological Spaces. Interscience Publishers, London-NY-Sydney.

[6] Tozzi, A. and Wyler, O. (1987) On Categories of Supertopological Spaces. Acta Universitatis Carolinae. Mathematica et Physica, 28, 137-149. 
[7] Banaschewski, B. (1964) Extensions of Topological Spaces. Canadian Mathematical Bulletin, 7, 1-23. http://dx.doi.org/10.4153/CMB-1964-001-5

[8] Bentley, H. (1975) Nearness Spaces and Extension of Topological Spaces. In: Studies in Topology, Academic Press, 47-66.

[9] Choquet, G. (1947) Sur les notions de fltre et de grille. Paris, Ser. A, C.R. Acad. Sci, 224, 171-173.

[10] Doîtchînov, D. (1985) Compactly Determined Extensions of Topological Spaces. SERDICA Bulgarice Math. Pub., 11, 269-286.

[11] Deák, J. (1993) Extending a Family of Screens in a Contiguity Space. Topology with Applications, 121-133.

[12] Kent, D. and MIN, W. (2002) Neighbourhood Spaces. IJMMS, 7, 387-399. 
Scientific Research Publishing (SCIRP) is one of the largest Open Access journal publishers. It is currently publishing more than 200 open access, online, peer-reviewed journals covering a wide range of academic disciplines. SCIRP serves the worldwide academic communities and contributes to the progress and application of science with its publication.

Other selected journals from SCIRP are listed as below. Submit your manuscript to us via either submit@scirp.org or Online Submission Portal.
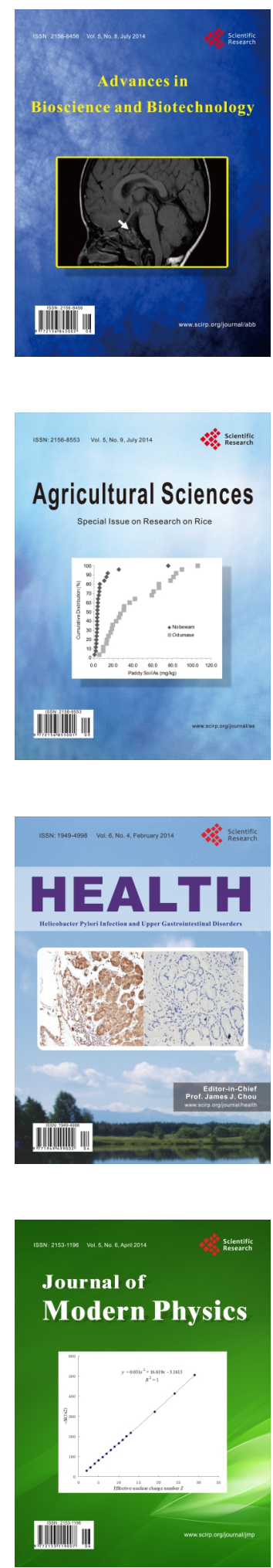
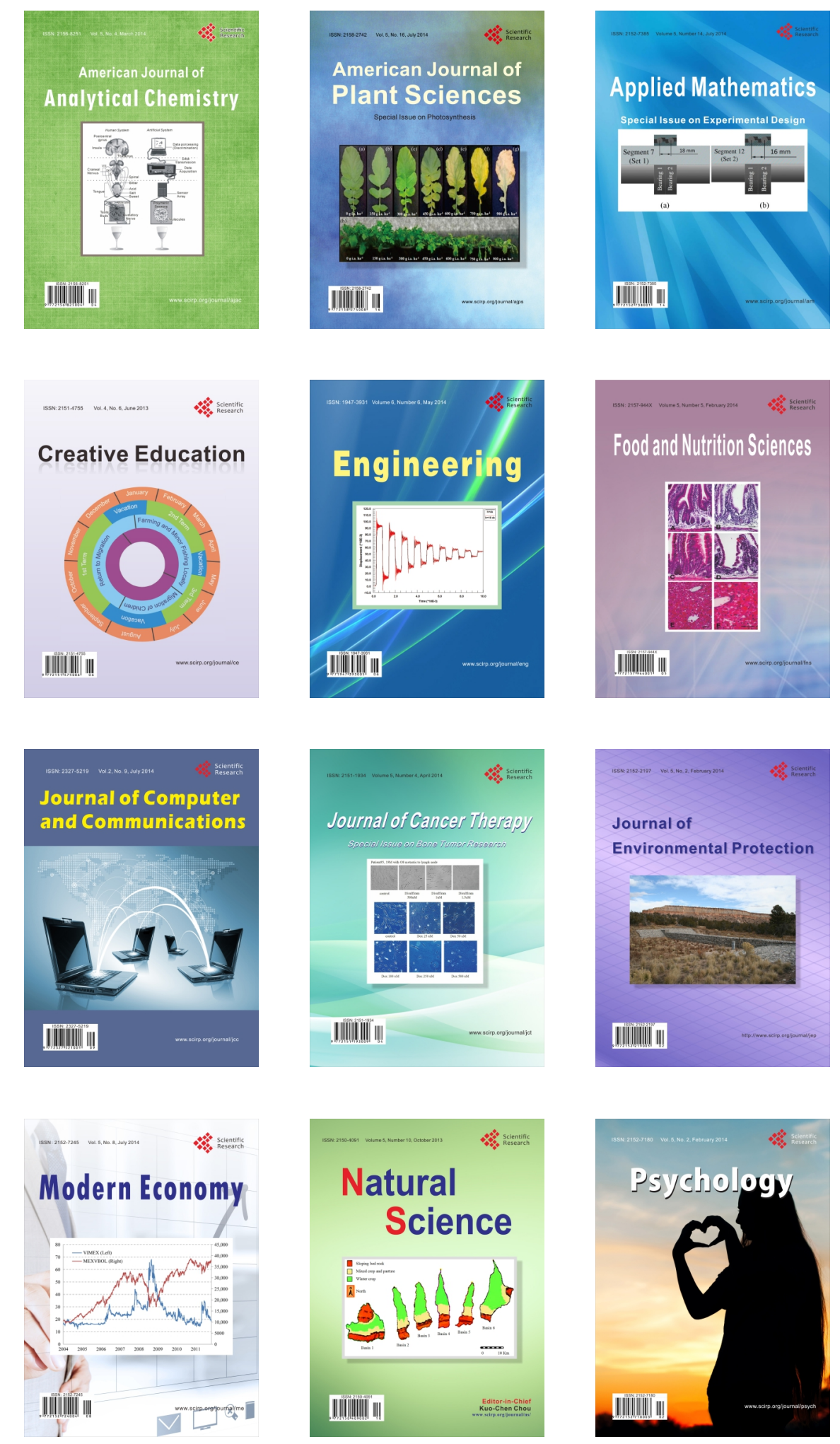